\title{
Connected Floer homology of covering involutions
}

\author{
Antonio Alfieri ${ }^{1}$ - Sungkyung Kang ${ }^{2,3} \cdot$ András I. Stipsicz $^{1}$ \\ Received: 30 July 2019 / Revised: 27 March 2020 / Published online: 28 April 2020 \\ (C) The Author(s) 2020
}

\begin{abstract}
Using the covering involution on the double branched cover of $S^{3}$ branched along a knot, and adapting ideas of Hendricks-Manolescu and Hendricks-Hom-Lidman, we define new knot (concordance) invariants and apply them to deduce novel linear independence results in the smooth concordance group of knots.
\end{abstract}

\section{Introduction}

Concordance questions of knots have been effectively studied by 4-dimensional topological methods by several authors. For a knot $K$ in the three-sphere $S^{3}$ one can consider the double branched cover $\Sigma(K)$ of $S^{3}$ branched along $K$. If $K$ is a slice knot (i.e. it bounds a smoothly embedded disk in the 4-disk $D^{4}$ ) then $\Sigma(K)$ bounds a four-manifold $X$ having the same rational homology as $D^{4}$ : this $X$ can be chosen to be the double branched cover of $D^{4}$ along the slice disk. The existence of such fourmanifold then can be obstructed by various methods, leading to sliceness obstructions of knots. For example, Donaldson's diagonalizability theorem applies in case $\Sigma(K)$ is known to bound a negative definite four-manifold with intersection form which does not embed into the same rank diagonal lattice. This line of reasoning was used by Lisca in his work about sliceness properties of 2-bridge knots, see [8,17,21]. A numerical

Communicated by Jean-Yves Welschinger.

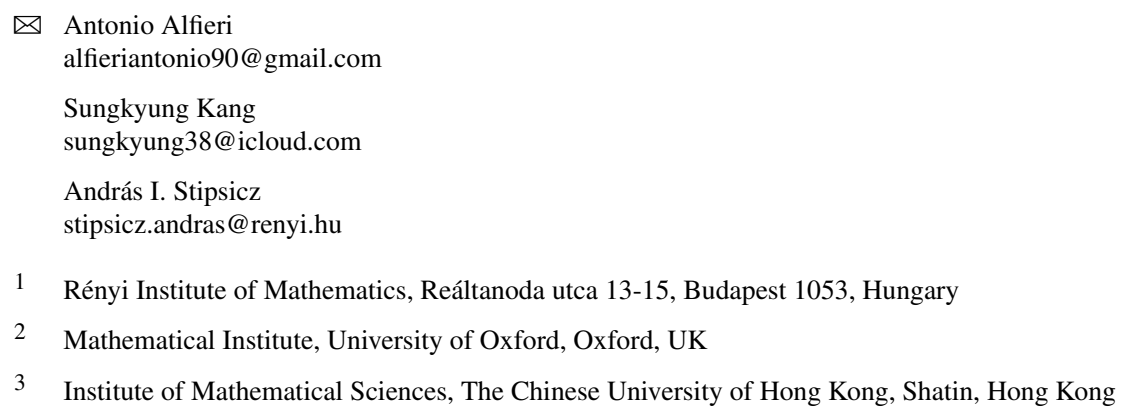


invariant (in the same spirit) was introduced by Manolescu-Owens [22] utilizing the Ozsváth-Szabó correction term of the unique spin structure of $\Sigma(K)$.

Different knots might admit diffeomorphic double branched covers, though; for example, if $K$ and $K^{\prime}$ differ by a Conway mutation, then $\Sigma(K)$ and $\Sigma\left(K^{\prime}\right)$ are diffeomorphic. This implies that if $K$ is slice, all slice obstructions coming from the above strategy must vanish for $K^{\prime}$ as well. A long-standing problem of this type was whether the Conway knot is slice; it admits a mutant (the Kinoshita-Terasaka knot) which is slice, hence merely considering the double branched cover will not provide sliceness obstruction. (The fact that the Conway knot is not slice has been recently proved by Piccirillo [36], relying on four-dimensional topological methods and results from Khovanov homology.)

The information we neglect in the above approach is that the three-manifold $\Sigma(K)$ (viewed as the double branched cover of $S^{3}$ along $K$ ) comes with a selfdiffeomorphism $\tau$, where pairs of points in $\Sigma(K)$ mapping to the same point of $S^{3}$ are interchanged by $\tau$. In this paper we introduce modifications of the usual Heegaard Floer homology groups of $\Sigma(K)$ which take this $\mathbb{Z} / 2 \mathbb{Z}$-action into account, leading to new knot invariants.

Heegaard Floer homology associates to a closed, oriented, smooth three-manifold a finitely generated $\mathbb{F}[U]$-module $\mathrm{HF}^{-}(Y)$ (where $\mathbb{F}[U]$ is the polynomial ring over the field $\mathbb{F}$ of two elements): it is the homology of a chain complex $\left(\mathrm{CF}^{-}(Y), \partial\right)$ (defined up to chain homotopy equivalence) and the homology naturally splits according to the $\operatorname{spin}^{c}$ structures of $Y$ as

$$
\operatorname{HF}^{-}(Y)=\bigoplus_{\mathfrak{s} \in \operatorname{Spin}^{c}(Y)} \operatorname{HF}^{-}(Y, \mathfrak{s}) .
$$

If $Y$ is a rational homology sphere (i.e., $\left.b_{1}(Y)=0\right)$ then $\operatorname{HF}^{-}(Y, \mathfrak{s})$ admits a natural $\mathbb{Q}$-grading, and the graded $\mathbb{F}[U]$-module $\operatorname{HF}^{-}(Y, \mathfrak{s})$ is a diffeomorphism invariant of the $\operatorname{spin}^{c}$ three-manifold $(Y, \mathfrak{s})$, while the local equivalence class of $\left(\mathrm{CF}^{-}(Y, \mathfrak{s}), \partial\right)$ (for the definition of this notion see Definition 3.2) provides an invariant of the rational $\operatorname{spin}^{c}$ homology cobordism class of $(Y, \mathfrak{s})$. In this case the local equivalence class of $\mathrm{CF}^{-}(Y, \mathfrak{s})$ can be characterised by a single rational number $d(Y, \mathfrak{s})$, the OzsváthSzabó correction term of the $\operatorname{spin}^{c}$ three-manifold $(Y, \mathfrak{s})$.

More recently, exploiting a symmetry built in the theory, Hendricks and Manolescu in [13] introduced involutive Heegaard Floer homology. The main idea of their construction is that, by making use of naturality, one can construct geometrically a grading-preserving chain map $\iota: \mathrm{CF}^{-}(Y) \rightarrow \mathrm{CF}^{-}(Y)$ which is (up to homotopy) an involution. The mapping cone of the map $\iota+$ id leads to involutive Floer homology $\operatorname{HFI}(Y)$, a module over the ring $\mathbb{F}[U, Q] /\left(Q^{2}\right)$. This group is interesting only for those $\operatorname{spin}^{c}$ structures which originate from a spin structure, and provides a new and rather sensitive diffeomorphism invariant of the underlying spin three-manifold. A further application of the above involution appeared in the work of Hendricks, Hom and Lidman [11], where (through the appropriate use of $\iota$ ) connected Heegaard Floer homology $\mathrm{HF}_{\text {conn }}^{-}(Y)$, a submodule of $\mathrm{HF}^{-}(Y)$ was defined. This submodule turned out to be a homology cobordism invariant. 
Similar constructions apply for any chain complex equipped with a homotopy involution. In this paper we will define the branched knot Floer homology of $K$ as $\operatorname{HFB}^{-}(K)=H_{*}\left(\operatorname{Cone}\left(\tau_{\#}+\mathrm{id}\right)\right)$, where $\tau: \Sigma(K) \rightarrow \Sigma(K)$ is the covering involution, $\tau_{\#}$ is the map induced by $\tau$ on the Heegaard Floer chain complex $\mathrm{CF}^{-}\left(\Sigma(K), \mathfrak{s}_{0}\right)$, with $\mathfrak{s}_{0}$ the unique $\operatorname{spin}^{c}$ structure on $\Sigma(K)$, and Cone is the mapping cone of the map $\tau_{\#}+$ id. (Related constructions have been examined in [12]; cf. also [2].)

Theorem 1.1 The group $\mathrm{HFB}^{-}(K)$, as a graded $\mathbb{F}[U, Q] /\left(Q^{2}\right)$-module, is an isotopy invariant of the knot $K \subset S^{3}$.

A simple argument shows that, as an $\mathbb{F}[U]$-module, $\operatorname{HFB}^{-}(K)$ is isomorphic to $\mathbb{F}[U]_{(\bar{\delta}(K))} \oplus \mathbb{F}[U]_{(\underline{\delta}(K))} \oplus A$, where $\bar{\delta}(K), \underline{\delta}(K) \in \mathbb{Q}$ and $A$ is a finitely generated, graded $U$-torsion module over $\mathbb{F}[U]$.

Theorem 1.2 The rational numbers $\bar{\delta}(K)$ and $\underline{\delta}(K)$ are knot concordance invariants.

Adapting the method of [11] for defining new homology cobordism invariants of rational homology spheres, we define the connected branched Floer homology $\mathrm{HFB}_{\text {conn }}^{-}(K)$ of a knot $K \subset S^{3}$ as follows. Consider a self-local equivalence $f_{\text {max }}: \mathrm{CF}^{-}\left(\Sigma(K), \mathfrak{s}_{0}\right) \rightarrow \mathrm{CF}^{-}\left(\Sigma(K), \mathfrak{s}_{0}\right)$ which commutes (up to homotopy) with $\tau_{\#}$ and has maximal kernel among such endomorphisms. Then take $\operatorname{HFB}_{\text {conn }}^{-}(K)=$ $H_{*}\left(\operatorname{Im} f_{\max }\right)$.

Theorem 1.3 The module $\mathrm{HFB}_{\mathrm{conn}}^{-}(K)$ (up to isomorphism) is independent of the choice of the map $f_{\max }$ with maximal kernel, and the isomorphism class of the graded module $\mathrm{HFB}_{\text {conn }}^{-}(K)$ is a concordance invariant of the knot $K$.

It follows from the construction that $\operatorname{HFB}_{\text {conn }}^{-}(K)$ is an $\mathbb{F}[U]$-submodule, indeed a direct summand of $\operatorname{HF}^{-}\left(\Sigma(K), \mathfrak{s}_{0}\right)$. As a finitely generated $\mathbb{F}[U]$-module, $\mathrm{HFB}_{\text {conn }}^{-}(K)$ is the sum of cyclic modules, and since it is of rank one, it can be written as

$$
\operatorname{HFB}_{\text {conn }}^{-}(K)=\mathbb{F}[U] \oplus \operatorname{HFB}_{\text {red-conn }}^{-}(K),
$$

where the second summand (the $U$-torsion submodule) is the reduced connected homology of $K$.

It is not hard to see that if $\mathrm{HF}^{-}\left(\Sigma(K), \mathfrak{s}_{0}\right)=\mathbb{F}[U]$ holds-for example if $\Sigma(K)$ is an $L$-space, which is the case when $K$ is a quasi-alternating knot-then $\tau_{\#}$ is chain homotopic to the identity map, implying the following.

Theorem 1.4 If $K$ is concordant to a quasi-alternating knot, then the reduced group $\mathrm{HFB}_{\text {red-conn }}^{-}(K)$ vanishes.

Somewhat more surprisingly, the same vanishing holds for torus knots (a behaviour similar to the one presented by the analogue of the upsilon-invariant defined in the seeting of Khovanov homology by Lewark and Lobb [19,27]):

Theorem 1.5 For the torus knot $T_{p, q}$ we have that $\mathrm{HFB}_{\text {red-conn }}^{-}\left(T_{p, q}\right)=0$. 
Finally we use lattice cohomology of Némethi [23] to get a computational scheme for the above invariants in the case of Montesinos knots. This second class of computation is based on results by Dai and Manolescu [4] and applies in particular to pretzel knots.

\subsection{Topological applications}

In [20] Lidman and Moore showed that a pretzel knot $P\left(a_{1}, \ldots, a_{k}\right)$ is an $L$-space knot (i.e. it has $L$-space surgeries) if and only if it is a torus knot of the form $T_{2,2 n+1}$, or a pretzel knot of the form $P(-2,3, q)$ for some $q \geq 7$ odd. In [39] the question of which linear combinations of $L$-space knots is concordant to a linear combination of algebraic knots was studied. In [1, Theorem 1.1] the first author showed that pretzel knots of the form $P(-2,3, q)$ are not concordant to positive sums of algebraic knots. It is conjectured that these knots are linearly independent in the quotient of the knot concordance group modulo algebraic knots. Note that for the pretzel knots $P(-2,3, q)$ the obstruction found in [39, Corollary 3.5] vanishes.

Our calculations for the connected homologies of pretzel knots in combination with the vanishing theorem above allow us to prove certain independence results in the smooth concordance group going in this direction. To state the results, let us introduce the following notations: let $\mathcal{C}$ denote the (smooth) concordance group of knots in $S^{3}$ and $\mathcal{Q} \mathcal{A}$ (respectively $\mathcal{T}$ ) those subgroups of $\mathcal{C}$ which are generated by all quasi-alternating (respectively torus) knots. In addition, $\mathcal{Q} \mathcal{A}+\mathcal{T}$ is the subgroup generated by quasi-alternating knots and torus knots.

Theorem 1.6 Let $K$ be the connected sum of pretzel knots of the form $P(-2,3, q)$, with $q \geq 7 \mathrm{odd}$. Then $K$ is not concordant to any linear combination of torus knots. Indeed, $[K]$ is a nonzero in the quotient $\mathcal{C} /(\mathcal{T}+\mathcal{Q A})$.

Furthermore, relying on computations from [4,15], and ideas and results from [3], we prove the following.

Theorem 1.7 The pretzel knots $\{P(-2 q-1,4 q+1,4 q+3) \mid q \geq 1\}$ are linearly independent in the quotient group $\mathcal{C} /(\mathcal{T}+\mathcal{Q} \mathcal{A})$. Indeed, $\mathbb{Z}^{\infty}$ is a direct summand of $\mathcal{C} /(\mathcal{T}+\mathcal{Q} \mathcal{A})$

Note that $P(-2 q-1,4 q+1,4 q+3)$ has trivial Alexander polynomial, thus is topologically slice. It was previously known that there exists an infinte family of topologically slice knots which is linearly independent in $\mathcal{C} / \mathcal{Q} \mathcal{A}$. Our result proves that we can also find such a family, not just up to quasi-alternating knots, but up to connected sums of quasi-alternating knots and torus knots. For independence from $L$-space knots, see [40].

\subsection{Organization of the paper}

The paper is organized as follows. In Sect. 2 we introduce branched knot Floer homology, and in Sect. 3 we discuss the details of the definition of the connected Floer 
homology group $\mathrm{HFB}_{\text {conn }}^{-}(K)$ of a knot $K \subset S^{3}$. Section 4 is devoted to the proof of the vanishing results above, while in Sect. 5 we give a way to compute the invariants for Montesinos knots. Finally in Sect. 6 we derive some independence results in the smooth concordance group.

We would like to thank András Némethi for numerous highly informative discussions. We specially thank the anonymous referee for providing a number of suggestions and advises which greatly improved the paper. The first and the third authors acknoweldge support from the NKFIH Élvonal project KKP126683. The second author was partially supported by the European Research Council (ERC) under the European Union Horizon 2020 research and innovation programme (grant agreement No 674978).

\section{Definition of branched knot Floer homology}

Let $\mathcal{H}=(\Sigma, \boldsymbol{\alpha}, \boldsymbol{\beta}, z)$ be a pointed Heegaard diagram which represents a rational homology sphere $Y$, and let $J_{s}$ be a generic path of almost-complex structures on the $g$ fold symmetric product $\operatorname{Sym}^{g}(\Sigma)$ (compatible with a symplectic structure constructed in [35]). Heegaard Floer homology [31] assigns to the pair $\left(\mathcal{H}, J_{s}\right)$ a finitely generated, $\mathbb{Q}$-graded chain complex $\mathrm{CF}^{-}\left(\mathcal{H}, J_{s}\right)$ over the polynomial ring $\mathbb{F}[U]$, graded so that $\operatorname{deg} U=-2$. This chain complex is defined as the free $\mathbb{F}[U]$-module generated by the intersection points of the Lagrangian tori $\mathbb{T}_{\alpha}=\alpha_{1} \times \cdots \times \alpha_{g}$ and $\mathbb{T}_{\beta}=\beta_{1} \times \cdots \times \beta_{g}$ in $\operatorname{Sym}^{g}(\Sigma)$, and is equipped with the differential

$$
\partial \mathbf{x}=\sum_{\mathbf{y} \in \mathbb{T}_{\alpha} \cap \mathbb{T}_{\beta}} \sum_{\left\{\phi \in \pi_{2}(\mathbf{x}, \mathbf{y}) \mid \mu(\phi)=1\right\}} \#\left(\frac{\mathcal{M}(\phi)}{\mathbb{R}}\right) U^{n_{z}(\phi)} \cdot \mathbf{y}
$$

where $\#(\mathcal{M}(\phi) / \mathbb{R})$ is the $(\bmod 2)$ number of points in the unparametrized moduli space $\mathcal{M}(\phi) / \mathbb{R}$ of $J_{s}$-holomorphic strips with index $\mu(\phi)=1$ representing the homotopy class $\phi \in \pi_{2}(\mathbf{x}, \mathbf{y})$, and $n_{z}(\phi)$ is the intersection number of $\phi$ with the divisor $V_{z}=$ $\{z\} \times \operatorname{Sym}^{g-1}(\Sigma)$. For more details about Heegaard Floer homology see [30-33].

For a knot $K \subset S^{3}$, let $\Sigma(K)$ denote the double branched cover of $S^{3}$ branched along $K$. The three-manifold $\Sigma(K)$ comes with a natural map $\tau: \Sigma(K) \rightarrow \Sigma(K)$ (called the covering involution) which interchanges points with equal image under the branched covering map $\pi: \Sigma(K) \rightarrow \Sigma(K) / \tau \simeq S^{3}$. The fixed point set $\operatorname{Fix}(\tau)=\widetilde{K}$ maps homeomorphically to $K$ under $\pi$. As the notation suggests ( since $H_{1}\left(S^{3} \backslash K ; \mathbb{Z}\right) \cong \mathbb{Z}$ ), the branched cover $\Sigma(K)$ in this case is determined by the branch locus $K \subset S^{3}$.

Pulling back the Heegaard surface, as well as the $\boldsymbol{\alpha}$ - and the $\boldsymbol{\beta}$-curves of a doublypointed Heegaard diagram $D=\left(\Sigma, \boldsymbol{\alpha}, \boldsymbol{\beta}, w_{1}, w_{2}\right)$ representing $K \subset S^{3}$, we get a pointed Heegaard diagram

$$
\mathcal{H}_{D}=\left(\widetilde{\Sigma}=\pi^{-1}(\Sigma), \widetilde{\boldsymbol{\alpha}}=\pi^{-1}(\boldsymbol{\alpha}), \widetilde{\boldsymbol{\beta}}=\pi^{-1}(\boldsymbol{\beta}), z=\pi^{-1}\left(w_{1}\right)\right)
$$

of the double branched cover $\Sigma(K)$. The covering projection $\pi: \Sigma(K) \rightarrow S^{3}$ restricts to a double branched cover of Riemann surfaces $\left.\pi\right|_{\widetilde{\Sigma}}: \widetilde{\Sigma} \rightarrow \Sigma$ with branch set 
$\left\{w_{1}, w_{2}\right\}$. The restriction $\left.\tau\right|_{\widetilde{\Sigma}}: \widetilde{\Sigma} \rightarrow \widetilde{\Sigma}$ of the covering involution $\tau$ represents the covering involution of $\left.\pi\right|_{\widetilde{\Sigma}}: \widetilde{\Sigma} \rightarrow \Sigma$, and $\left.\tau\right|_{\widetilde{\Sigma}}: \widetilde{\Sigma} \rightarrow \widetilde{\Sigma}$ induces a self-diffeomorphism of the symmetric product

$$
\sigma: \operatorname{Sym}^{\tilde{g}}(\widetilde{\Sigma}) \rightarrow \operatorname{Sym}^{\tilde{g}}(\widetilde{\Sigma}),
$$

leaving $\mathbb{T}_{\widetilde{\alpha}}$ and $\mathbb{T}_{\widetilde{\beta}}$, as well as the divisor $V_{z}=\{z\} \times \operatorname{Sym}^{\tilde{g}-1}(\widetilde{\Sigma})$ invariant.

Pick a generic path of almost-complex structures $J_{S} \in \mathcal{J}\left(\operatorname{Sym}^{\tilde{g}}(\widetilde{\Sigma})\right.$ ) (satisfying the usual compatibility conditions with the chosen symplectic form on the symmetric product) and consider the Heegaard Floer chain complex $\mathrm{CF}^{-}\left(\mathcal{H}_{D}, J_{S}\right)$ associated to $\left(\mathcal{H}_{D}, J_{S}\right)$. Recall that there is a direct sum decomposition of $\mathrm{CF}^{-}\left(\mathcal{H}_{D}, J_{S}\right)$ indexed by $\operatorname{spin}^{c}$ structures:

$$
\mathrm{CF}^{-}\left(\mathcal{H}_{D}, J_{S}\right)=\bigoplus_{\mathfrak{s} \in \operatorname{Spin}^{\mathrm{c}}(\Sigma(K))} \mathrm{CF}^{-}\left(\mathcal{H}_{D}, J_{s} ; \mathfrak{s}\right)
$$

The first (singular) homology group of the double branched cover $\Sigma(K)$ can be presented by $\theta+\theta^{t}$, where $\theta$ is a Seifert matrix for $K$. Thus, $\left|H_{1}(\Sigma(K), \mathbb{Z})\right|=$ $\operatorname{det}\left(\theta+\theta^{\prime}\right)=\operatorname{det}(K)$, which is an odd number. In particular, $\Sigma(K)$ has a unique spin structure $\mathfrak{s}_{0}$. We will focus on $\mathrm{CF}^{-}\left(\mathcal{H}_{D}, J_{s} ; \mathfrak{s}_{0}\right)$, the summand of $\mathrm{CF}^{-}\left(\mathcal{H}_{D}, J_{S}\right)$ associated to $\mathfrak{s}_{0}$.

Note that if the path of almost-complex structures $J_{S} \in \mathcal{J}\left(\operatorname{Sym}^{\tilde{g}}(\widetilde{\Sigma})\right)$ is chosen generically, transversality is achieved for both $J_{s}$ and the push-forward $\sigma_{*} J_{S}$ (where $\sigma$ is given in Equation (2)). For such a choice of almost-complex structures we have well-defined Heegaard Floer chain complexes $\mathrm{CF}^{-}\left(\mathcal{H}_{D}, J_{S}\right)$ and $\mathrm{CF}^{-}\left(\mathcal{H}_{D}, \sigma_{*} J_{S}\right)$, and we can consider the map

$$
\eta: \mathrm{CF}^{-}\left(\mathcal{H}_{D}, J_{S}\right) \rightarrow \mathrm{CF}^{-}\left(\mathcal{H}_{D}, \sigma_{*} J_{s}\right)
$$

sending a generator $\mathbf{x}=x_{1}+\cdots+x_{\tilde{g}} \in \mathbb{T}_{\widetilde{\alpha}} \cap \mathbb{T}_{\widetilde{\beta}} \subset \operatorname{Sym}^{\tilde{g}}(\widetilde{\Sigma})$ to $\sigma(\mathbf{x})=\tau\left(x_{1}\right)+$ $\cdots+\tau\left(x_{\tilde{g}}\right)$.

Lemma 2.1 The map $\eta$ is an isomorphism of chain complexes. Furthermore, $\eta$ maps the summand $\mathrm{CF}^{-}\left(\mathcal{H}_{D}, J_{s} ; \mathfrak{s}_{0}\right)$ of the spin structure $\mathfrak{s}_{0}$ into $\mathrm{CF}^{-}\left(\mathcal{H}_{D}, \sigma_{*} J_{s} ; \mathfrak{s}_{0}\right)$.

Proof It is obviously an isomorphism of free $\mathbb{F}[U]$-modules; indeed, $\eta^{2}=$ id since $\tau$ is an involution. To see that $\eta$ commutes with the differential, notice that $u \mapsto \sigma \circ u$ provides a diffeomorphism between the moduli space of $J_{S}$-holomorphic representatives of a homotopy class $\phi \in \pi_{2}(\mathbf{x}, \mathbf{y})$ and the moduli space of $\sigma_{*} J_{S}$-holomorphic representatives of $\left.\sigma \circ \phi \in \pi_{2}(\sigma(\mathbf{x}), \sigma(\mathbf{y}))\right)$.

To show that $\eta$ preserves the spin structure we argue as follows. According to $\left[31\right.$, Sect. 2.6] the choice of a basepoint $z$ of $\mathcal{H}_{D}$ determines a map $\mathfrak{s}_{z}: \mathbb{T}_{\widetilde{\alpha}} \cap \mathbb{T}_{\widetilde{\beta}} \rightarrow$ $\operatorname{Spin}^{\mathrm{c}}(\Sigma(K))$ and

$$
\mathrm{CF}^{-}\left(\mathcal{H}_{D}, J_{s} ; \mathfrak{s}\right)=\bigoplus_{\mathfrak{s}_{z}(\mathbf{x})=\mathfrak{s}} \mathbb{F}[U] \cdot \mathbf{x}
$$


It follows from the definition of $\mathfrak{s}_{z}$ that $\tau_{*}\left(\mathfrak{s}_{z}(\mathbf{x})\right)=\mathfrak{s}_{z}(\tau(\mathbf{x}))$. Thus if $\mathfrak{s}_{z}(\mathbf{x})=\mathfrak{s}_{0}$, we have that

$$
\overline{\mathfrak{s}_{z}(\tau(\mathbf{x}))}=\overline{\tau_{*}\left(\mathfrak{s}_{z}(\mathbf{x})\right)}=\overline{\tau_{*}\left(\mathfrak{s}_{0}\right)}=\tau_{*}\left(\overline{\mathfrak{s}_{0}}\right)=\tau_{*}\left(\mathfrak{s}_{0}\right)=\mathfrak{s}_{z}(\tau(\mathbf{x}))
$$

proving that $\mathfrak{s}_{z}(\tau(\mathbf{x}))$ is a self-conjugate $\operatorname{spin}^{c}$ structure, i.e. spin. The claim now follows from the fact that $\Sigma(K)$ has a unique spin structure.

We define $\tau_{\#}: \mathrm{CF}^{-}\left(\mathcal{H}_{D}, J_{S} ; \mathfrak{s}_{0}\right) \rightarrow \mathrm{CF}^{-}\left(\mathcal{H}_{D}, J_{s} ; \mathfrak{s}_{0}\right)$ as the map $\eta: \mathrm{CF}^{-}\left(\mathcal{H}_{D}, J_{s} ; \mathfrak{s}_{0}\right) \rightarrow \mathrm{CF}^{-}\left(\mathcal{H}_{D}, \sigma_{*} J_{s} ; \mathfrak{s}_{0}\right)$ followed by the continuation map

$$
\Phi_{J_{s, t}}^{-}: \mathrm{CF}^{-}\left(\mathcal{H}_{D}, \sigma_{*} J_{s} ; \mathfrak{s}_{0}\right) \rightarrow \mathrm{CF}^{-}\left(\mathcal{H}_{D}, J_{s} ; \mathfrak{s}_{0}\right)
$$

from [31, Sect. 6], induced by a generic two-parameter family $J_{s, t}$ of almost-complex structures interpolating between $J_{s}$ and $\tau_{*} J_{s}$ :

$$
\tau_{\#}=\Phi_{J_{s, t}}^{-} \circ \eta
$$

Lemma $2.2 \tau_{\#}^{2} \simeq \mathrm{id}$, where $\simeq$ denotes chain homotopy equivalence.

Proof Consider

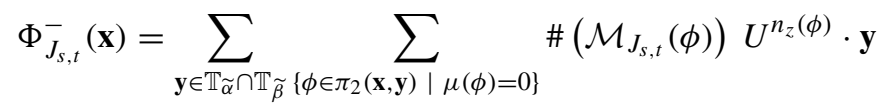

where $\mathcal{M}_{J_{s, t}}(\phi)$ denotes the moduli spaces of $J_{s, t}$-holomorphic strips.

Given $\mathbf{x} \in \mathbb{T}_{\widetilde{\alpha}} \cap \mathbb{T}_{\widetilde{\beta}}$ one computes

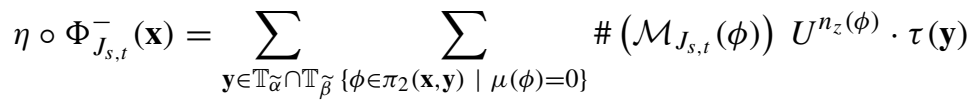

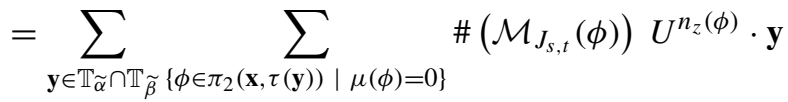

$$
\begin{aligned}
& =\sum_{\mathbf{y} \in \mathbb{T}_{\widetilde{\alpha}} \cap \mathbb{T}_{\widetilde{\beta}}} \sum_{\left\{\phi \in \pi_{2}(\tau(\mathbf{x}), \mathbf{y}) \mid \mu(\phi)=0\right\}} \#\left(\mathcal{M}_{J_{s, t}}(\tau \circ \phi)\right) U^{n_{z}(\sigma \circ \phi)} \cdot \mathbf{y} \\
& =\sum_{\mathbf{y} \in \mathbb{T}_{\tilde{\alpha}} \cap \mathbb{T}_{\tilde{\beta}}} \sum_{\left\{\phi \in \pi_{2}(\tau(\mathbf{x}), \mathbf{y}) \mid \mu(\phi)=0\right\}} \#\left(\mathcal{M}_{J_{s, t}}(\phi)\right) U^{n_{z}(\phi)} \cdot \mathbf{y} \\
& =\Phi_{\tau_{*} J_{s, t}}^{-}(\tau(\mathbf{x})) \text {, }
\end{aligned}
$$

hence the identity $\eta \circ \Phi_{J_{s, t}}^{-}=\Phi_{\sigma_{*} J_{s, t}}^{-} \circ \eta$ follows. Thus,

$$
\tau_{\#}^{2}=\Phi_{J_{s, t}}^{-} \circ \eta \circ \Phi_{J_{s, t}}^{-} \circ \eta=\Phi_{J_{s, t}}^{-} \circ \Phi_{\sigma_{*} J_{s, t}}^{-} \circ \eta^{2}=\Phi_{J_{s, t}}^{-} \circ \Phi_{\sigma_{*} J_{s, t}}^{-},
$$


where the last identity holds because $\eta^{2}=$ id (a consequence of the fact that $\tau: \Sigma(K) \rightarrow \Sigma(K)$ is an involution).

By concatenating $J_{s, t}$ and $\sigma_{*} J_{s, t}$ we obtain a one-parameter family of paths of almost-complex structures describing a closed loop based at the path $J_{s}$. Since the space of almost complex structures (compatible with the fixed symplectic structure) is contractible, we can find a three-parameter family of almost complex structures $J_{s, t, x}$ interpolating between the juxtaposition of $J_{s, t}$ and $\sigma_{*} J_{s, t}$, and $J_{s, t, 1} \equiv J_{s}$. As pointed out in [31, Sect. 6], a generic choice of $J_{s, t, x}$ produces smooth moduli spaces

$$
\mathcal{M}_{J_{s, t, x}}(\phi)=\bigcup_{c \in[0,1]} \mathcal{M}_{J_{s, t, c}}(\phi) \quad \phi \in \pi_{2}(\mathbf{x}, \mathbf{y})
$$

of dimension $\mu(\phi)+1$. These can be used to produce a chain homotopy equivalence

$$
H_{J_{s, t, x}}^{-}(\mathbf{x})=\sum_{\mathbf{y} \in \mathbb{T}_{\widetilde{\alpha}} \cap \mathbb{T}_{\widetilde{\beta}}\left\{\phi \in \pi_{2}(\mathbf{x}, \mathbf{y}) \mid \mu(\phi)=-1\right\}} \#\left(\mathcal{M}_{J_{s, t, x}}(\phi)\right) U^{n_{z}(\phi)} \cdot \mathbf{y}
$$

between $\Phi_{J_{s, t}}^{-} \circ \Phi_{\sigma_{*} J_{s, t}}^{-}$and id, concluding the argument.

In summary, for a knot $K \subset S^{3}$ there is a homotopy involution $\tau_{\#}: \mathrm{CF}^{-}\left(\Sigma(K), \mathfrak{s}_{0}\right)$ $\rightarrow \mathrm{CF}^{-}\left(\Sigma(K), \mathfrak{s}_{0}\right)$ associated to the covering involution $\tau: \Sigma(K) \rightarrow \Sigma(K)$. In order to derive knot invariants from the pair $\left(\mathrm{CF}^{-}\left(\mathcal{H}, \mathfrak{s}_{0}\right), \tau_{\#}\right)$, we follow ideas from [13] and form the mapping cone of $\tau_{\#}+\mathrm{id}: \mathrm{CF}^{-}\left(\Sigma(K), \mathfrak{s}_{0}\right) \rightarrow \mathrm{CF}^{-}\left(\Sigma(K), \mathfrak{s}_{0}\right)$, written equivalently as

$$
\mathrm{CFB}^{-}(K)=\left(\mathrm{CF}^{-}\left(\Sigma(K), \mathfrak{s}_{0}\right)[-1] \otimes \mathbb{F}[Q] /\left(Q^{2}\right), \partial_{\text {cone }}=\partial+Q \cdot\left(\tau_{\#}+\mathrm{id}\right)\right),
$$

where $\operatorname{deg} Q=-1$. Recall that $\mathrm{CF}^{-}\left(\Sigma(K), \mathfrak{s}_{0}\right)$ admits an absolute $\mathbb{Q}$-grading, and

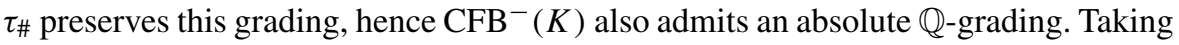
homology we get the group $\mathrm{HFB}^{-}(K)=H_{*}\left(\mathrm{CFB}^{-}(K)\right)$, which is now a module over the ring $\mathbb{F}[U, Q] /\left(Q^{2}\right)$. We call $\mathrm{HFB}^{-}(K)$ the branched Heegaard Floer homology of the knot $K \subset S^{3}$. Now we are ready to turn to the proof of the first statement announced in Sect. 1:

Proof of Theorem 1.1 The proof is similar to the one of [13, Proposition 2.8]. Independence from the chosen path of almost-complex structures is standard Floer theory. For independence from the chosen doubly pointed Heegaard diagram of $K$, we argue as follows: A doubly pointed Heegaard diagram $D=\left(\Sigma, \boldsymbol{\alpha}, \boldsymbol{\beta}, w_{1}, w_{2}\right)$ representing the knot $K \subset S^{3}$ can be connected to any other doubly pointed diagram $D^{\prime}=\left(\Sigma^{\prime}, \boldsymbol{\alpha}^{\prime}, \boldsymbol{\beta}^{\prime}, w_{1}^{\prime}, w_{2}^{\prime}\right)$ of $K$ by a sequence of isotopies and handleslides of the $\boldsymbol{\alpha}$-curves (or $\boldsymbol{\beta}$-curves) supported in the complement of the two basepoints, and by stabilizations (i.e., forming the connected sum of $\Sigma$ with a torus $T^{2}$ equipped with a new pair of curves $\alpha_{g+1}$ and $\beta_{g+1}$ which meet transversally in a single point). A sequence of these moves lifts to a sequence of pointed Heegaard moves of the pullback diagrams $\mathcal{H}_{D}$ and $\mathcal{H}_{D^{\prime}}$ with underlying three-manifold the double branched cover 
$\Sigma(K)$. According to [16] the choice of such a sequence of Heegaard moves yields a natural chain homotopy equivalence $\psi: \mathrm{CF}^{-}\left(\mathcal{H}_{D}\right) \rightarrow \mathrm{CF}^{-}\left(\mathcal{H}_{D^{\prime}}\right)$ which fits into the diagram

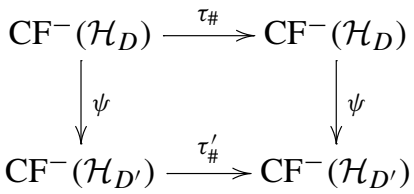

that commutes up to chain homotopy. Let $\Gamma: \mathrm{CF}^{-}\left(\mathcal{H}_{D}\right) \rightarrow \mathrm{CF}^{-}\left(\mathcal{H}_{D^{\prime}}\right)$ be a map realizing the chain homotopy equivalence. Then the map $f: \operatorname{Cone}\left(\tau_{\#}+\mathrm{id}\right) \rightarrow$ Cone $\left(\tau_{\#}^{\prime}+\mathrm{id}\right)$ defined by $f=\psi+Q \cdot(\psi+\Gamma)$ is a quasi-isomorphism. Indeed, $f$ is a filtered map with respect to the two step filtration of the mapping cones, and since it induces an isomorphism on the associated graded objects, it is a quasi-isomorphism.

Notice that the mapping cone exact sequence associated to $\mathrm{CFB}^{-}(K)=\mathrm{Cone}\left(\tau_{\#}+\right.$ id) reads as an exact triangle

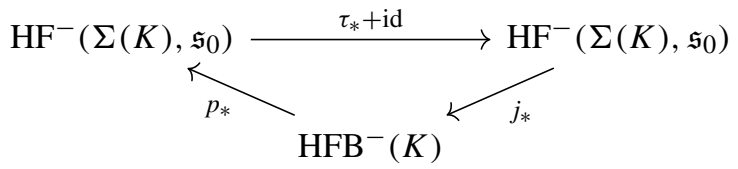

in which $j_{*}$ preserves the grading, and $p_{*}$ drops it by one. In particular, if $\tau_{*}=\mathrm{id}$, the horizontal map in the above triangle is zero, and in that case $\mathrm{HFB}^{-}(K)$ is the sum of two copies of $\mathrm{HF}^{-}\left(\Sigma(K), \mathfrak{s}_{0}\right)$ (with the grading on one copy shifted by one). have

A close inspection of the exact triangle above reveals that, as $\mathbb{F}[U]$-modules, we

$$
\operatorname{HFB}^{-}(K)=\mathbb{F}[U]_{(\bar{\delta})} \oplus \mathbb{F}[U]_{(\underline{\delta}+1)} \oplus(\mathbb{F}[U] \text {-torsion })
$$

We set $\underline{\delta}(K)=\underline{\delta}$ and $\bar{\delta}(K)=\bar{\delta}$, which (by the above discussion) are knot invariants. Notice that $\underline{\delta}(K), \bar{\delta}(K) \in \mathbb{Q}, \underline{\delta}(K) \equiv \delta(K) \equiv \bar{\delta}(K) \bmod 2$, and $\underline{\delta}(K) \leq \delta(K) \leq$ $\bar{\delta}(K)$, where $\delta(K)$ is the Ozsváth-Szabó correction term of $\left(\Sigma(K), \mathfrak{s}_{0}\right)$, i.e. $\delta(K)$ is half the Manolescu-Owens invariant of $K$ introduced in [22].

\section{Concordance invariants from $\left(\mathrm{CF}^{-}\left(\Sigma(K), \mathfrak{s}_{0}\right), \tau_{\#}\right)$}

Adapting ideas from [11], the chain complex $\mathrm{CF}^{-}\left(\Sigma(K), \mathfrak{s}_{0}\right)$, equipped with $\tau_{\#}$, provides concordance invariants of the knot $K$ as follows. First, recall [11, Definition 2.5] regarding $\iota$-complexes:

Definition 3.1 An $\iota$-complex $(C, \iota)$ is a finitely generated, free, $\mathbb{Q}$-graded chain complex $C$ over $\mathbb{F}[U]$ together with a map $\iota: C \rightarrow C$ where $C$ is supported in degree $d+\mathbb{Z}$ 
for some $d \in \mathbb{Q}$ (multiplication by $U$ drops the $\mathbb{Q}$-grading by two), the homology of the localization $U^{-1} H_{*}(C)=H_{*}\left(C \otimes_{\mathbb{F}[U]} \mathbb{F}\left[U, U^{-1}\right]\right)$ is isomorphic to $\mathbb{F}\left[U, U^{-1}\right]$ via an isomorphism preserving the relative $\mathbb{Z}$-grading, and $\iota$ is a grading preserving, $U$-equivariant chain map which is a homotopy involution.

We will consider $\iota$-complexes up to local equivalence (see [11, Definitions 2.6 and 2.7]).

Definition 3.2 A local equivalence $f: C \rightarrow C^{\prime}$ of two $\iota$-complexes $(C, \iota)$ and $\left(C^{\prime}, \iota^{\prime}\right)$ is a grading preserving, $U$-equivariant chain map $f: C \rightarrow C^{\prime}$ such that

- $\iota^{\prime} \circ f \simeq f \circ \iota$, i.e. the two compositions are chain homotopy equivalent, and

- $f$ induces an isomorphisms $f_{\text {loc }}$ on the localization $U^{-1} H_{*}(C)$.

Definition 3.3 Two $\iota$-complexes $(C, \iota)$ and $\left(C^{\prime}, \iota^{\prime}\right)$ are locally equivalent if there exist local equivalences $f: C \rightarrow C^{\prime}$ and $g: C^{\prime} \rightarrow C$. If in addition we have $f \circ g \simeq$ id and $g \circ f \simeq \mathrm{id}$, then $(C, \iota)$ and $\left(C^{\prime}, \iota^{\prime}\right)$ are chain homotopy equivalent $\iota$-complexes.

Given an $\iota$-complex $(C, \iota)$, we can look at the set $\operatorname{End}_{\text {loc }}(C, \iota)$ of its self-local equivalences $f: C \rightarrow C$. This can be partially ordered by defining $f \preceq g$ if and only if $\operatorname{Ker} f \subset \operatorname{Ker} g$. We say that $f \in \operatorname{End}_{\text {loc }}(C)$ is a maximal self-local equivalence if it is maximal with respect to this ordering. Since we assumed $C$ to be finitely generated, maximal self-local equivalences always exist. The following lemma summarises the results of [11, Section 3].

Lemma 3.4 Let $(C, \iota)$ be an ı-complex. Then

(1) if $f \in \operatorname{End}_{\text {loc }}(C, \iota)$ is a maximal self-local equivalence, then ı induces a homotopy involution $\iota^{\operatorname{Im} f}: \operatorname{Im} f \rightarrow \operatorname{Im} f$. Furthermore, $\left(\operatorname{Im} f, \iota^{\operatorname{Im} f}\right)$ is locally equivalent to $(C, \iota)$;

(2) if $f, h \in \operatorname{End}_{\text {loc }}(C, \iota)$ are two maximal self-local equivalences, then there is a chain homotopy equivalence $\left(\operatorname{Im} f, \iota^{\operatorname{Im} f}\right) \simeq\left(\operatorname{Im} h, \iota^{\operatorname{Im} h}\right)$ of $\iota$-complexes;

(3) if $\left(C^{\prime}, \iota^{\prime}\right)$ is an $\iota$-complex locally equivalent to $(C, \iota)$ and $f \in \operatorname{End}_{10 c}(C, \iota)$, and $h \in \operatorname{End}_{\mathrm{loc}}\left(C^{\prime}, \iota^{\prime}\right)$ are self-local equivalences then there is a chain homotopy equivalence $\left(\operatorname{Im} f, \iota^{\operatorname{Im} f}\right) \simeq\left(\operatorname{Im} h, \iota^{\operatorname{Im} h}\right)$ of $\iota$-complexes.

Since

$$
H_{*}\left(\mathrm{CF}^{-}\left(\Sigma(K), \mathfrak{s}_{0}\right) \otimes_{\mathbb{F}[U]} \mathbb{F}\left[U, U^{-1}\right]\right)=\operatorname{HF}^{\infty}\left(\Sigma(K), \mathfrak{s}_{0}\right)=\mathbb{F}\left[U, U^{-1}\right],
$$

the pair $\left(\mathrm{CF}^{-}\left(\Sigma(K), \mathfrak{s}_{0}\right), \tau_{\#}\right)$ of the Heegaard Floer chain complex of the double branched cover $\Sigma(K)$ of a knot $K \subset S^{3}$ (equipped with the homotopy involution $\tau_{\#}$ induced by the covering involution) is an $\iota$-complex associated to $K$. Given a maximal self-local equivalence $f_{\max }: \mathrm{CF}^{-}\left(\Sigma(K), \mathfrak{s}_{0}\right) \rightarrow \mathrm{CF}^{-}\left(\Sigma(K), \mathfrak{s}_{0}\right)$ we define $\mathrm{HFB}_{\text {conn }}^{-}(K)$, the connected Floer homology of the knot $K \subset S^{3}$ as $H_{*}\left(\operatorname{Im} f_{\max }\right)$. As an application of Lemma 3.4, it is then easy to see that the resulting group, up to isomorphism, is a knot invariant: 
Theorem 3.5 The chain homotopy type of $\operatorname{Im} f_{\max }$ is independent of the choice of the maximal self-local equivalence $f_{\max } \in \operatorname{End}_{\text {loc }}\left(\mathrm{CF}^{-}\left(\Sigma(K), \mathfrak{s}_{0}\right), \tau_{\#}\right)$, hence $\operatorname{HFB}_{\text {conn }}^{-}(K)$ is a knot invariant.

We now turn to the proof of the concordance invariance of the groups $\mathrm{HFB}_{\text {conn }}^{-}(K)$. The following naturality statement will be crucial in the proof.

Lemma 3.6 (Ozsváth \& Szabó [34], Zemke [41]) Let $Y$ and $Y^{\prime}$ be two three-manifolds equipped with self-diffeomorphisms $\tau: Y \rightarrow Y$ and $\tau^{\prime}: Y^{\prime} \rightarrow Y^{\prime}$. Suppose that $W: Y \rightarrow Y^{\prime}$ is a cobordism and that there exists a self-diffeomorphism $T: W \rightarrow W$ restricting to $\tau$ and $\tau^{\prime}$ on the two ends of $W$. Then

$$
\tau_{\#}^{\prime} \circ F_{W, \mathfrak{t}}^{-}=F_{W, T_{*} \mathrm{t}}^{-} \circ \tau_{\#}
$$

maps $\mathrm{CF}^{-}\left(Y,\left.\mathfrak{t}\right|_{Y}\right) \rightarrow \mathrm{CF}^{-}\left(Y^{\prime},\left.\mathfrak{t}\right|_{Y^{\prime}}\right)$ for every $\mathfrak{t} \in \operatorname{Spin}^{\mathrm{c}}(W)$.

Proof of Theorem 1.3 Suppose that $K^{\prime} \subset S^{3}$ is concordant to $K$, i.e. there exists a smoothly embedded annulus $C \subset S^{3} \times[0,1]$ with $\partial C=C \cap S^{3} \times[0,1]=K \times$ $\{1\} \cup K^{\prime} \times\{0\}$. By taking the double branched cover $\Sigma(C)$ of $S^{3} \times[0,1]$ branched along $C$ we get a smooth rational homology cobordism from $\Sigma\left(K^{\prime}\right)$ to $\Sigma(K)$. By adapting [10, Lemma 2.1] for $n=2$, we get that the four-manifold $\Sigma(C)$ comes with a distinguished spin structure $\mathfrak{t}$ restricting to the canonical spin structure on the two ends. In addition, this spin structure is invariant under the covering involution of the double branched cover $\Sigma(C)$. Let $F_{C}^{-}: \mathrm{CF}^{-}\left(\Sigma(K), \mathfrak{s}_{0}\right) \rightarrow \mathrm{CF}^{-}\left(\Sigma\left(K^{\prime}\right), \mathfrak{s}_{0}\right)$ denote the cobordism map induced by $(\Sigma(C), \mathfrak{t})$.

Since $\Sigma(C)$ is a rational homology cobordism, it follows that

$$
F_{C}^{-}: \mathrm{CF}^{-}\left(\Sigma(K), \mathfrak{s}_{0}\right) \rightarrow \mathrm{CF}^{-}\left(\Sigma\left(K^{\prime}\right), \mathfrak{s}_{0}\right)
$$

and

$$
F_{-C}^{-}: \mathrm{CF}^{-}\left(\Sigma\left(K^{\prime}\right), \mathfrak{s}_{0}\right) \rightarrow \mathrm{CF}^{-}\left(\Sigma(K), \mathfrak{s}_{0}\right)
$$

are local equivalences. (Recall that according to [34], a rational homology cobordism induces an isomorphism on $\mathrm{HF}^{\infty}=U^{-1} \mathrm{HF}^{-}$.) The fact that $F_{C}^{-}$and $F_{-C}^{-}$both homotopy commute with the homotopy $\mathbb{Z} / 2 \mathbb{Z}$-actions follows from Lemma 3.6 and the fact that (in the notations of that lemma) we have $T_{*} \mathrm{t}=\mathfrak{t}$. Then Lemma 3.4 concludes the argument.

Proof of Theorem 1.2 Let $f \in \operatorname{End}_{\text {loc }}\left(\mathrm{CF}^{-}\left(\Sigma(K), \mathfrak{s}_{0}\right), \tau_{\#}\right)$ be a maximal self-local equivalence. As a consequence of Lemma 3.4, the chain homotopy type of the mapping cone of the restriction $\tau_{\#}^{\operatorname{Im} f}+\mathrm{id}: \operatorname{Im} f \rightarrow \operatorname{Im} f$ is a concordance invariant of $K$. On the other hand,

$$
H_{*}\left(\operatorname{Cone}\left(\tau_{\#}^{\operatorname{Im} f}+\mathrm{id}\right)\right)=\mathbb{F}[U]_{\underline{\delta}(K)} \oplus \mathbb{F}[U]_{\bar{\delta}(K)+1} \oplus(\mathbb{F}[U]-\text { torsion }),
$$

implying the claim. 
Since $\mathrm{HF}^{-}\left(\Sigma(K), \mathfrak{s}_{0}\right)$ is of rank one (as an $\mathbb{F}[U]$-module), and $f_{\max }$ is a local equivalence, it follows that the summand $\operatorname{HFB}_{\text {conn }}^{-}(K) \subset \mathrm{HF}^{-}\left(\Sigma(K), \mathfrak{s}_{0}\right)$ is also of rank one. The $U$-torsion submodule of $\operatorname{HFB}_{\text {conn }}^{-}(K)$ is the reduced connected Floer homology of $K$, and it will be denoted by $\operatorname{HFB}_{\text {red-conn }}^{-}(K)$. The following simple adaptation of [11, Proposition 4.6] allows us to prove triviality of $\operatorname{HFB}_{\text {red-conn }}^{-}(K)$.

Proposition 3.7 $\mathrm{HFB}_{\text {red-conn }}^{-}(K)=0$ if and only if $\bar{\delta}(K)=\underline{\delta}(K)=\delta(K)$.

Given a knot $K \subset S^{3}$ we denote by $-K$ its mirror image.

Lemma 3.8 For a knot $K \subset S^{3}$ we have that $\underline{\delta}(K)=-\bar{\delta}(-K)$.

Proof The double branched cover of $-K$ is $-\Sigma(K)$. The argument of [13, Proposition 5.2] provides the claimed identity.

Lemma 3.9 If $K=K_{1} \# K_{2}$ for two knots $K_{1}$ and $K_{2} \subset S^{3}$, then

$$
\underline{\delta}\left(K_{1}\right)+\underline{\delta}\left(K_{2}\right) \leq \underline{\delta}(K) \leq \bar{\delta}(K) \leq \bar{\delta}\left(K_{1}\right)+\bar{\delta}\left(K_{2}\right) \text {. }
$$

Proof Suppose that $D_{i}$ is a doubly pointed Heegaard diagram for $K_{i} \subset S^{3}(i=1,2)$. Then a doubly pointed Heegaard diagram $D$ can be constructed for $K$ by taking the connected sums of $D_{i}$ (along $w_{2}^{1}$ in $D_{1}$ and $w_{2}^{2}$ in $D_{2}$ ). Then a simple argument shows that the Heegaard diagram $\mathcal{H}_{D}$ of $\Sigma(K)$ originating from $D$ can be given as the connected sum of $\mathcal{H}_{D_{1}}$ and $\mathcal{H}_{D_{2}}$, implying that $\mathrm{CF}^{-}\left(\mathcal{H}_{D}\right)$ is the tensor product of $\mathrm{CF}^{-}\left(\mathcal{H}_{D_{1}}\right)$ and $\mathrm{CF}^{-}\left(\mathcal{H}_{D_{2}}\right)$. It follows that the map $\eta_{D}$ of Equation (3) for $\mathcal{H}_{D}$ is the tensor product of the similar maps $\eta_{D_{1}}$ and $\eta_{D_{2}}$ for $\mathcal{H}_{D_{1}}$ and $\mathcal{H}_{D_{2}}$. Since the map induced on $\mathrm{CF}^{-}$by Heegaard moves on $\mathcal{H}_{D_{1}}$ and $\mathcal{H}_{D_{2}}$ splits as the tensor product of maps induced on $\mathrm{CF}^{-}\left(\mathcal{H}_{D_{1}}\right)$ and $\mathrm{CF}^{-}\left(\mathcal{H}_{D_{2}}\right)$ respectively (note that this fact was also used by Hendricks-Manolescu-Zemke in the proof of [14, Theorem 1.1]), we see that $\left(\tau_{D}\right)_{\#}$ and $\left(\tau_{D_{1}}\right)_{\#} \otimes\left(\tau_{D_{2}}\right)_{\#}$ are chain homotopic maps, from which a simple adaptation of the proof of [14, Proposition 1.3] implies the result.

\section{Vanishing results}

In some cases $\mathrm{HFB}^{-}(K)$ and $\mathrm{HFB}_{\text {conn }}^{-}(K)$ can be easily determined. As is customary in Heegaard Floer theory, these invariants do not capture any new information for quasi-alternating knots. It is a more surprising (and as we will see, very convenient) feature of $\mathrm{HFB}_{\mathrm{conn}}^{-}$that it is rather trivial for torus knots as well. In this section we show some vanishing results about the group $\operatorname{HFB}_{\text {red-conn }}^{-}(K)$, while the next section provides methods to determine our invariants for Montesinos (and more generally for arborescent) knots. We start with a simple motivating example.

\subsection{An example}

Consider the Brieskorn sphere $\Sigma(2,3,7)$; it can be given as $(-1)$-surgery on the right-handed trefoil knot $T_{2,3}$. It is an integral homology sphere with Heegaard Floer 
homology $\widehat{\mathrm{HF}}(\Sigma(2,3,7))=\mathbb{F}_{(0)}^{2} \oplus \mathbb{F}_{(-1)}$ and $\mathrm{HF}^{-}(\Sigma(2,3,7))=\mathbb{F}[U]_{(-2)} \oplus \mathbb{F}_{(-2)}$ in its unique $\operatorname{spin}^{c}$ (hence spin) structure, see [28, Eq. (25)].

This three-manifold can be presented as the double branched cover of $S^{3}$ either along the torus knot $T_{3,7}$, or along the pretzel knot $P(2,-3,-7)$. The two presentations provide two involutions on $\Sigma(2,3,7)$, which potentially provide two different maps on the Heegaard Floer chain complex. Indeed, let $\phi_{1}$ denote the involution $\Sigma(2,3,7)$ admits as double branched cover along $T_{3,7}$ and let $\phi_{2}$ denote the involution it gets as double branched cover along $P(2,-3,-7)$. Through direct calculation, the actions of these maps on Heegaard Floer homology has been identified in [12, Propositions 6.26 and 6.27].

Theorem 4.1 ( [12]) The map $\left(\phi_{1}\right)_{*}$ induces the identity map on $\widehat{\mathrm{HF}}(\Sigma(2,3,7))$ (and hence on $\mathrm{HF}^{-}(\Sigma(2,3,7))$, while the map $\left(\phi_{2}\right)_{*}$ is different from the identity on $\widehat{\mathrm{HF}}(\Sigma(2,3,7))$.

This allows us to compute the invariants $\mathrm{HFB}^{-}$and $\mathrm{HFB}_{\mathrm{conn}}^{-}$for $T_{3,7}$ and $P(2,-3,-7)$, showing that

- $\operatorname{HFB}^{-}\left(T_{3,7}\right)=\mathbb{F}[U]_{(-2)} \oplus \mathbb{F}[U]_{(-3)} \oplus \mathbb{F}_{(-2)} \oplus \mathbb{F}_{(-3)}$, or, as an $\mathbb{F}[U, Q] /\left(Q^{2}\right)$ module (and ignoring gradings) $\mathrm{HFB}^{-}\left(T_{3,7}\right)=\left(\mathbb{F}[U, Q] /\left(Q^{2}\right)\right) \oplus \mathbb{F}^{2}$,

- $\operatorname{HFB}_{\text {conn }}^{-}\left(T_{3,7}\right)=\mathbb{F}[U]_{(-2)}$, hence $\operatorname{HFB}_{\text {red-conn }}^{-}\left(T_{3,7}\right)=0$;

- $\operatorname{HFB}^{-}(P(2,-3,-7))=\left(\mathbb{F}[U, Q] /\left(Q^{2}\right)\right) \oplus \mathbb{F}$,

- $\operatorname{HFB}_{\text {conn }}^{-}(P(2,-3,-7))=\mathbb{F}[U]_{(-2)} \oplus \mathbb{F}_{(-2)}$, hence $\operatorname{HFB}_{\text {red-conn }}^{-}(P(2,-3,-7))$ $=\mathbb{F}_{(-2)} \neq 0$.

These calculations generalize to show that any torus knot has trivial reduced connected Floer homology $\mathrm{HFB}_{\text {red-conn }}^{-}$, while for pretzel knots there is a combinatorial method to determine this quantity. In particular, the above results will be reproved in Sect. 4.3 and in Sect. 6.

\subsection{Quasi-alternating knots}

Proof of Theoem 1.4 If $K$ is a quasi-alternating knot, then the double branched cover $\Sigma(K)$ is an $L$-space, and hence $\mathrm{HF}^{-}\left(\Sigma(K), \mathfrak{s}_{0}\right)=\mathbb{F}[U]$; in particular the homology is only in even degrees. Results of [4] imply that $\tau_{\#}$ is determined (up to homotopy) by its action on homology, which (as a grading preserving map) for $\mathbb{F}[U]$ must be equal to the identity. Therefore $\tau_{\#}$ is homotopic to the identity, and so $\tau_{\#}+\mathrm{id}=0$, hence the exact triangle of Eq. (5) determines $\mathrm{HFB}^{-}(K)$ as the sum of two copies of $\mathrm{HF}^{-}\left(\Sigma(K), \mathfrak{s}_{0}\right)$ (one with shifted grading). Furthermore, since the homotopy commuting assumption of a self-local equivalence in this case is vacuous, we get that $\operatorname{HFB}_{\text {conn }}^{-}(K)=\mathbb{F}[U](=$ $\left.\mathrm{HF}^{-}\left(\Sigma(K), \mathfrak{s}_{0}\right)\right), \mathrm{HFB}_{\text {red-conn }}^{-}(K)=0$, and the only invariant we get from this picture is the $d$-invariant of $\left(\Sigma(K), \mathfrak{s}_{0}\right)$, which is (half of) the Manolescu-Owens invariant of the knot $K$ from [22].

\subsection{Torus knots}

Next we turn to the discussion of Theorem 1.5. Thanks to the observation below, the proof of this result is significantly easier when $p q$ is odd. 
Proposition 4.2 Suppose that pq is odd. Then the covering involution $\tau$ on the double branched cover $\Sigma\left(T_{p, q}\right)$ is isotopic to id.

Proof The double branched cover of the torus knot $T_{p, q}$ is diffeomorphic to the link of the complex surface singularity given by the equation $z^{2}=x^{p}+y^{q}$, which is the Brieskorn sphere $\Sigma(2, p, q)$. The covering involution $\tau: \Sigma(2, p, q) \rightarrow \Sigma(2, p, q)$ of $T_{p, q}$ acts as $(z, x, y) \mapsto(-z, x, y)$.

Fix $t \in S^{1}$ and consider the diffeomorphism

$$
(z, x, y) \mapsto\left(t^{p q} z, t^{2 q} x, t^{2 p} y\right)
$$

Clearly we get an $S^{1}$-family of diffeomorphisms, where $t=1$ gives id, while $t=-1$ (under the condition $p q$ odd) gives $\tau$, concluding the proof of the proposition.

The case when exactly one of $p$ and $q$ is even, however, requires some more preparation from lattice homology. (For a more thorough introduction to this subject see $[23,25])$.

\subsection{Lattice homology}

This theory was motivated by computational results of Ozsváth and Szabó (for manifolds given by negative definite plumbing trees of at most one 'bad' vertex) in [29] and extended by Némethi [23] to any negative definite plumbing trees. The isomorphism of lattice homology with Heegaard Floer homology was established for almost-rational graphs by Némethi in [23], which was extended in [26] to graphs with at most two 'bad' vertices. Below we recall the basic concepts and results of this theory.

A graded root is a pair $(R, w)$ where

- $R$ is a directed, infinite tree with a finite number of leaves and a unique end modelled on the infinite stem , subject to the condition that every vertex has exactly one successor (see Fig. 1 for an example),

- and $w: R \rightarrow \mathbb{Q}$ is a function associating to each vertex $x$ of $R$ a rational number $w(x) \in \mathbb{Q}$ such that $w\left(x_{2}\right)=w\left(x_{1}\right)-2$ if $\left(x_{1}, x_{2}\right)$ is an edge of $R$.

A graded root $(R, w)$ specifies a graded $\mathbb{F}[U]$-module $\mathbb{H}^{-}(R, w)$ as follows: As a graded vector space, $\mathbb{H}^{-}(R, w)$ is generated over $\mathbb{F}$ by the vertices of $R$, graded so that $\operatorname{gr}(x)=w(x)$ for all $x \in R$. Multiplication by $U$ is defined on the set of generators by saying that $U \cdot x=y$ if for the vertex $x$ of $R$ the vertex $y$ is its successor.

Manolescu and Dai showed in [4] that the lattice homology $\mathbb{H}^{-}\left(R, w_{k}\right)$ corresponding to the graded root $\left(R, w_{k}\right)$ can be represented as the homology of a model complex $C(R)$, which is defined as follows. Let $C(R)$ be generated (as an $\mathbb{F}[U]$-module), by

- the leaves $\left\{v_{l}\right\}$ of $R$ (with grading $w\left(v_{l}\right)$ ), called the even generators, and

- by the odd generators defined as follows: for a vertex $a$ of $R$ with valency greater than 2 , let $V=\left\{v_{1}, \ldots, v_{n}\right\}$ be the set of all vertices of $R$ satisfying $U v=a$. Then we take the formal sums $v_{1}-v_{2}, \ldots, v_{n-1}-v_{n}$ of vertices of $R$ as generators of $C(R)$ at degree $w(a)+1$. 
Fig. 1 A graded root

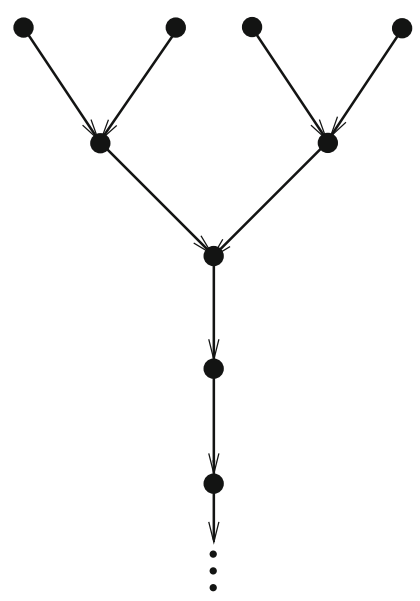

The $U$-action in this module lowers degree by 2 . The differential $\partial$ on $C(R)$ vanishes on all even generators $v$, while for an odd generator $a$ the definition is slightly more complicated. Let $v$ and $w$ be two even generators (i.e. leaves of $R$ ) such that $a=$ $U^{m} v-U^{n} w$ as formal sums of vertices of $R$, for some nonnegative integers $m$ and $n$. Then set $\partial(a)=U^{m+1} v-U^{n+1} w$. In this case we say that the odd generator $a$ is an angle between the even generators $v$ and $w$ (For pictorial descriptions of $C(R)$, see [4]).

A negative definite plumbing tree $\Gamma$ (with associated plumbed four-manifold $X_{\Gamma}$ ) and a characteristic vector $k$ of $H^{2}\left(X_{\Gamma}, \mathbb{Z}\right)$ determines a graded root $\left(R_{\Gamma}, w_{k}\right)$ as follows. Let $L$ be the non-compact 1-dimensional $C W$-complex having the points of $H_{2}\left(X_{\Gamma}, \mathbb{Z}\right)=\mathbb{Z}^{|\Gamma|}$ as 0 -cells and a 1 -cell connecting two vertices $\ell, \ell^{\prime} \in H_{2}\left(X_{\Gamma} ; \mathbb{Z}\right)$ if $\ell^{\prime}=\ell+v$ for some $v \in \Gamma$. The characteristic vector $k \in H^{2}\left(X_{\Gamma}, \mathbb{Z}\right)=$ $\operatorname{Hom}\left(H_{2}\left(X_{\Gamma}\right), \mathbb{Z}\right)$ determines a quadratic function $\chi_{k}: H_{2}\left(X_{\Gamma} ; \mathbb{Z}\right) \rightarrow \mathbb{Z}$ through the formula

$$
\chi_{k}(\ell)=-\frac{1}{2}\left(k(\ell)+\ell^{2}\right) .
$$

For each $n \in \mathbb{Z}$ let $S_{n}$ be the set of connected components of the subcomplex of $L$ spanned by the vertices satisfying $\chi_{k} \leq n$. We define $R_{\Gamma}$ to be the graph with vertex set $\bigcup_{n \geq 0} S_{n}$, in which two vertices $x_{1}$ and $x_{2}$ are connected by a directed edge if and only if the elements $x_{1} \in S_{n}$ and $x_{2} \in S_{n+1}$ (corresponding to components of the sublevel sets $\chi_{k} \leq n$ and $\chi_{k} \leq n+1$, respectively) satisfy $x_{1} \subset x_{2}$. We define $w_{k}: R_{\Gamma} \rightarrow \mathbb{Q}$ for $x \in S_{n}$ by the formula

$$
w_{k}(x)=\frac{k^{2}+|\Gamma|}{4}-2 n .
$$

A negative definite plumbing graph $\Gamma$ is rational if it is the resolution graph of a rational singularity, i.e. a singularity with geometric genus $p_{g}=0$. According to [24, Theorem 1.3] a negative definite graph is rational if and only if the boundary $Y_{\Gamma}$ of the 
associated four-dimensional plumbing $X_{\Gamma}$ is a Heegaard Floer $L$-space. We say that $\Gamma$ is almost-rational if it is negative definite, and there is a vertex $v$ of $\Gamma$ on which we can change the weight in such a way that the result is rational.

Theorem 4.3 ( [29]) Let $\Gamma$ be an almost-rational graph, $\mathfrak{s} \in \operatorname{Spin}^{c}\left(Y_{\Gamma}\right)$ a spin ${ }^{c}$ structure on $Y_{\Gamma}$, and $k$ a characteristic vector of the intersection lattice of $X_{\Gamma}$ representing a spinc structure that restricts to $\mathfrak{s}$ on the boundary $\partial X_{\Gamma}=Y_{\Gamma}$. Then there is an isomorphism $\mathbb{H}^{-}\left(R_{\Gamma}, w_{k}\right) \simeq \operatorname{HF}^{-}\left(Y_{\Gamma}, \mathfrak{s}\right)$ of graded $\mathbb{F}[U]$-modules.

\subsection{Torus knots again}

With this preparation in place, we now return to the computation of invariants of torus knots. We start by describing a plumbing presentation of $\Sigma\left(T_{p, q}\right)$, where the covering involution $\tau$ is also visible. In what follows $p$ and $q$ denote two coprime, positive integers with $p q$ even.

Lemma 4.4 The double branched cover $\Sigma\left(T_{p, q}\right)$ of $S^{3}$ branched along the torus knot $T_{p, q}$ can be presented by a plumbing graph $\Gamma_{p, q}$ with the following properties:

- the graph $\Gamma_{p, q}$ is star-shaped with three legs $L_{f i x}, L_{1}, L_{2}$ going out from the central vertex $c$,

- the coefficients on $L_{1}$ and $L_{2}$ are the same,

- the covering involution $\tau$ of $\Sigma\left(T_{p, q}\right)$ extends smoothly to an involution $T$ of the plumbed four-manifold $X_{p, q}$ associated to $\Gamma_{p, q}$. More precisely we can take $T$ to be the map fixing the portion of $X_{p, q}$ corresponding to the arm $L_{f i x}$, and flipping the portion corresponding to $L_{1}$ and $L_{2}$.

Proof Recall that the double branched cover $\Sigma\left(T_{p, q}\right)$ is equal to the link of the hypersurface singularity $z^{2}=x^{p}+y^{q}$, hence $\Sigma\left(T_{p, q}\right)$ can be presented as a plumbed manifold along the resolution dual graph of the above singularity. This graph can be easily determined by computing first the embedded resolution of the curve singularity $x^{p}+y^{q}=0$, and then using a simple algorithm (described, for example in [7, Sect. 7.2]) for computing the resolution graph of the singularity. The embedded resolution of $T_{p, q}$ gives a linear graph, where the single $(-1)$-curve is intersected by the proper transform of the knot, and the multiplicities at the two ends are $p$ and $q$, respectively (see [5]). Now [7, Lemma 7.2.8] shows that if say $p$ is even, then the curves between the leaf with multiplicity $p$ and the $(-1)$-curve intersecting the proper transform have all even multiplicities. The algorithm described in [7, Sect. 7.2] then provides the resolution graph, together with the information about the covering transformation, satisfying the properties listed in the lemma.

We denote by $R_{p, q}$ the graded root associated to $\Gamma_{p, q}$ as in Sect. 4.4. Recall that in computing a graded root, we have to choose a characteristic vector $k \in H^{2}\left(X_{\Gamma} ; \mathbb{Z}\right)$, so that we can work with the induced weight function $\chi_{k}$. Here, we choose $k$ to be the canonical characteristic vector, which is given by $k(v)=-2-v^{2}$, where $v^{2}$ denotes the self-intersection of the vertex $v$ in the plumbing graph.

The map $T: X_{p, q} \rightarrow X_{p, q}$ of Lemma 4.4 induces an involution $T_{*}$ of the graded module $\mathbb{H}^{-}\left(R_{p, q}, \chi_{k}\right)$. Indeed, $T$ induces an involution of the lattice $H_{2}\left(X_{p, q} ; \mathbb{Z}\right) \subset$ 
$H_{2}\left(X_{p, q} ; \mathbb{R}\right)$ that preserves the weights assigned according to $\chi_{k}$. The following was observed in [4, Remark 4.3].

Lemma 4.5 If $(R, w)$ is a graded root, then a grading preserving homomorphism $f: \mathbb{H}^{-}(R, w) \rightarrow \mathbb{H}^{-}(R, w)$ lifts, uniquely up to chain homotopy, to a grading preserving chain map $f_{\#}: C(R) \rightarrow C(R)$. In addition, if $C$ is a free chain complex with $H_{*}(C) \simeq \mathbb{H}^{-}(R, w)$ then $C \simeq C(R)$.

Thus the involution $T_{*}: \mathbb{H}^{-}\left(R_{p, q}, \chi_{k}\right) \rightarrow \mathbb{H}^{-}\left(R_{p, q}, \chi_{k}\right)$ lifts to an involution $T_{\#}$ : $C_{*}\left(R_{p, q}\right) \rightarrow C_{*}\left(R_{p, q}\right)$ of the model complex associated to the graded root $R_{p, q}$.

Lemma $4.6\left(C_{*}\left(R_{p, q}\right), T_{\#}\right)$ is chain homotopy equivalent to $\left(\mathrm{CF}^{-}\left(\Sigma\left(T_{p, q}\right), \mathfrak{s}_{0}\right), \tau_{\#}\right)$.

Proof The canonical class $k$ is clearly $\mathbb{Z} / 2 \mathbb{Z}$-invariant, so the induced $\operatorname{spin}^{c}$ structure $\left.[k]\right|_{\partial X_{p, q}} \in \operatorname{Spin}^{c}\left(\Sigma\left(T_{p, q}\right)\right)$ on the boundary is also $\mathbb{Z} / 2 \mathbb{Z}$-invariant. Recall that the $\mathbb{Z} / 2 \mathbb{Z}$-action on the set of $\operatorname{spin}^{c}$ structures on $\Sigma\left(T_{p, q}\right)$ leaves the unique spin structure $\mathfrak{s}_{0}$ invariant, and indeed $\mathfrak{s}_{0}$ is the unique fixed point of the action, as shown in [9, Page 1378] and [18, Remark 3.4]. Hence the lattice homology of $\Gamma_{p, q}$ with respect to $k$ computes $\mathrm{HF}^{-}\left(\Sigma\left(T_{p, q}\right), \mathfrak{s}_{0}\right)$.

In order to extend the isomorphism to the connected groups, as a consequence of Lemma 4.5, it is sufficient to prove that $\tau_{*}: \operatorname{HF}^{-}\left(\Sigma\left(T_{p, q}\right), \mathfrak{s}_{0}\right) \rightarrow \operatorname{HF}^{-}\left(\Sigma\left(T_{p, q}\right), \mathfrak{s}_{0}\right)$ and $T_{*}: \mathbb{H}^{-}\left(R_{p, q}, \chi_{k}\right) \rightarrow \mathbb{H}^{-}\left(R_{p, q}, \chi_{k}\right)$ correspond to each other under the isomorphism $\mathbb{H}^{-}\left(R_{p, q}, \chi_{k}\right) \simeq \operatorname{HF}^{-}\left(\Sigma\left(T_{p, q}\right), \mathfrak{s}_{0}\right)$.

Unravelling the definition of the isomorphism of Theorem 4.3, as it was done in [4, Theorem 3.1], the claim boils down to the identity

$$
\tau_{\# \circ F_{X_{p, q}, \xi}^{-}}=F_{X_{p, q}, T_{*} \xi}^{-},
$$

where $\xi=k+2 \cdot \operatorname{PD}(\ell)$, with $\ell \in H_{2}\left(X_{p, q}, \mathbb{Z}\right)$ any characteristic vector restricting to the only spin structure $\mathfrak{s}_{0}$ on $\partial X_{p, q}=\Sigma\left(T_{p, q}\right)$. This identity on the other hand is exactly the content of Lemma 3.6.

Lemma 4.7 The reduced connected homology associated to $\left(C_{*}\left(R_{p, q}\right), T_{\#}\right)$ vanishes.

Proof First, we claim that there exists a $\mathbb{Z} / 2 \mathbb{Z}$-invariant vertex $v \in V\left(R_{k}\right)$ with minimal $\chi_{k}$-value. To prove this, we have to find a lattice point $x=\sum x_{v} \cdot v \in \mathbb{Z}^{|\Gamma|}$ which satisfies the following properties:

- $x$ is $\mathbb{Z} / 2 \mathbb{Z}$-invariant, i.e. the coefficients of $x$ on the $\operatorname{arm} L_{1}$ and the coefficients of $x$ on $L_{2}$ are the same.

- $\chi_{k}(x) \leq \chi_{k}(y)$ for any other lattice point $y=\sum_{v \in V(\Gamma)} y_{v} \cdot v$.

Once we have found such a lattice point, the claim about the vertex $v$ can be proved using the following argument. Recall that vertices of $R_{k}$ are components of sublevel sets of $\chi_{k}$. The $\mathbb{Z} / 2 \mathbb{Z}$-action on $R_{k}$ permutes the components of sublevel sets, hence if we denote the component of the minimally-weighted sublevel set which contains the invariant lattice point $x$ by $C$, then $C$ is fixed by the $\mathbb{Z} / 2 \mathbb{Z}$-action. 
To see the existence of $x$ with the above properties, we first choose any lattice point $x_{0}=\sum_{v \in V(\Gamma}\left(x_{0}\right)_{v} \cdot v$ such that $\chi_{k}\left(x_{0}\right)$ is minimal. Then we can write $\chi_{k}\left(x_{0}\right)$ as

$$
\chi_{k}\left(x_{0}\right)=\chi_{k}\left(x_{0}^{f i x}\right)+Q_{1}\left(x_{0}\right)+Q_{2}\left(x_{0}\right),
$$

where $x_{0}^{f i x}=\sum_{v \in L_{f i x} \cup c} x_{v} \cdot v$ is the "fixed part" of $x_{0}$ and $Q_{1}, Q_{2}$ are functions defined on $\mathbb{Z}^{|\Gamma|}$ using the formula

$$
-2 Q_{i}(x)=\sum_{v \in L_{i}}\left(x_{v}^{2} \cdot e_{v}+x_{v} k_{v}\right)+\sum_{\text {edge }\left(v_{1} v_{2}\right) \text { in } L_{i}} x_{v_{1}} x_{v_{2}}+x_{v_{c}^{i}} x_{c}
$$

for $i=1,2$. (Here, we have denoted the vertex in $L_{i}$ which is connected to the central vertex $c$ as $v_{c}^{i}$.)

Assume, without loss of generality, that $Q_{1}\left(x_{0}\right) \leq Q_{2}\left(x_{0}\right)$, and consider the lattice point $x^{\prime}$ defined as

$$
x^{\prime}=x_{0}^{f i x}+\sum_{v \in V\left(L_{1}\right)} x_{v} \cdot(v+\sigma(v))
$$

where $\sigma$ denotes the $\mathbb{Z} / 2 \mathbb{Z}$-action on $\Gamma$ coming from the symmetry found in Lemma 4.4. Then we have

$$
\chi_{k}\left(x^{\prime}\right)=\chi_{k}\left(x_{0}^{f i x}\right)+2 Q_{1}\left(x_{0}\right) \leq \chi_{k}\left(x_{0}\right)+Q_{1}\left(x_{0}\right)+Q_{2}\left(x_{0}\right)=\chi_{k}\left(x_{0}\right) .
$$

Since we assumed that $\chi_{k}\left(x_{0}\right)$ is minimal among all lattice points, we get $\chi_{k}\left(x^{\prime}\right)=$ $\chi_{k}\left(x_{0}\right)$, implying $x^{\prime}$ satisfies the desired properties.

Now we claim that our symmetric graded root $R=R_{k}$ is locally equivalent to another symmetric graded root $R^{\prime}$, where the $\mathbb{Z} / 2 \mathbb{Z}$-action on $R^{\prime}$ is trivial. This can be verified by induction on the number $n_{R}$ of non- $\mathbb{Z} / 2 \mathbb{Z}$-invariant leaves of $R$. In this part of the proof, in the notation we will confuse graded roots with their associated model complexes. For example, when we write that two given symmetric graded roots are locally equivalent, we will actually mean that their associated model chain complexes are locally equivalent.

The base case is simple: if $n_{R}=0$, then the $\mathbb{Z} / 2 \mathbb{Z}$-action on $R$ is already trivial, so we are done. In the general case, choose a non- $\mathbb{Z} / 2 \mathbb{Z}$-invariant leaf $v$ of $R$. Since $R$ carries a $\mathbb{Z} / 2 \mathbb{Z}$-invariant leaf in its top-degree level, we can always find a $\mathbb{Z} / 2 \mathbb{Z}$ invariant vertex $x$ of $R$ which lies in the same grading as $v$ does. Denote the angle between the infinite monotone path starting at $v$ and at $x$ by $\alpha$. Then we define $R_{1}$ as the graded root associated to the model complex we get from the model complex of $R$ by deleting $v, \sigma(v)$ and the angles $\alpha, \sigma(\alpha)$. Define a map $F$ from the associated model complex of $R$ to that of $R_{1}$ as follows.

- $F(v)=F(\sigma(v))=x$ and $F(w)=w$ for any leaf $w \neq v$.

- $F(\alpha)=F(\sigma(\alpha))=0$ and $F(\beta)=\beta$ for any angle $\beta \neq \alpha$.

- Extend this map $\mathbb{F}[U]$-linearly to the model chain complex of $R$. 

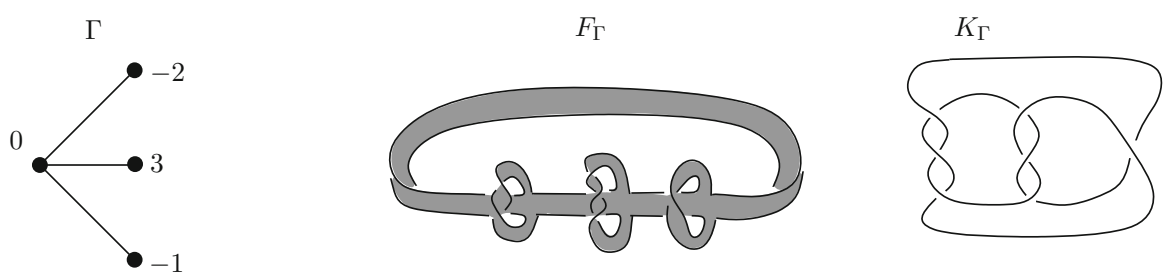

Fig. 2 The plumbing graph $\Gamma$ on the left determines a surface $F_{\Gamma}$ (shown in the middle) with boundary $K_{\Gamma}$ (on the right)

This map $F$ is obviously $\mathbb{F}[U]$-linear and $\mathbb{Z} / 2 \mathbb{Z}$-equivariant, so if we only prove that $F$ is a chain map, it would automatically be a local equivalence to its image. To check that $F$ is a chain map, it suffices to check that $\partial(F(v))=F(\partial v)$ and $\partial(F(\alpha))=F(\partial \alpha)$ by linearity and equivariance. Indeed,

- $\partial(F(v))=\partial($ even generator $)=0$ and $F(\partial v)=F(0)=0$,

- $\partial(F(\alpha))=\partial(0)=0$ and $F(\partial \alpha)=F(v+x)=F(v)+F(x)=x+x=0$.

Consequently $F$ is a local equivalence. This implies that $R$ is locally equivalent to $R_{1}$, and the number of non- $\mathbb{Z} / 2 \mathbb{Z}$-invariant leaves of $R_{1}$ is strictly smaller than the number of non- $\mathbb{Z} / 2 \mathbb{Z}$-invariant leaves of $R$. Thus, by induction, we deduce that $R$ is locally equivalent to a symmetric graded root $R^{\prime}$ whose $\mathbb{Z} / 2 \mathbb{Z}$-action is trivial. This gives us the equality $\bar{\delta}(K)=\underline{\delta}(K)$, which by Proposition 3.7 then implies the claim.

Proof of Theorem 1.5 When $p q$ is odd, Proposition 4.2 shows that the covering transformation on $\Sigma\left(T_{p, q}\right)$ is homotopic to id, implying the vanishing of the reduced connected homology. For $p q$ even the combination of Lemmas 4.4 and 4.7 implies that $\mathrm{HFB}_{\text {red-conn }}^{-}\left(T_{p, q}\right)=0$, concluding the proof.

\section{Arborescent and montesinos knots}

A plumbing tree $\Gamma$ is a tree whose vertices are labelled by integers (see $\Gamma$ on Figure 2 below). Above we associated a four-manifold $X_{\Gamma}$ (and its three-dimensional boundary $Y_{\Gamma}$ ) to a plumbing tree $\Gamma$. A variant of this construction associates a surface $F_{\Gamma} \subset S^{3}$ to $\Gamma$ : for every vertex we consider an annulus or a Möbius band, given by introducing half-twists dictated by the label of the vertex, and plumb (i.e. Murasugi sum) these annuli and Möbius bands together according to $\Gamma$. (See Fig. 2 for a simple example.) Following [6, Definition 1.3], the boundary of $F_{\Gamma}$ specifies a link $K_{\Gamma}=\partial F_{\Gamma}$, called an arborescent link associated to $\Gamma$.

Remark 5.1 Notice that the link is not determined uniquely by the graph, since at vertices of higher valency we need to determine an order for the edges when considering a planar presentation, and the link might depend on this choice. In addition, the location of the plumbing region relative to the twists also might influence the resulting link. With slightly more information (see Gabai's introductory work in [6]) attached to the tree this procedure can be made unique, though. 
The resulting $K_{\Gamma}$ is called a Montesinos link if the tree $\Gamma$ is star-shaped; it is a pretzel link if $\Gamma$ is star-shaped with all legs of length one, and is two-bridge if the graph $\Gamma$ is linear.

The construction of the four-manifold and the knot associated to $\Gamma$ is connected by the fact that (by repeated application of Montesinos' trick) the double branched cover $\Sigma\left(K_{\Gamma}\right)$ of an arborescent link $K_{\Gamma}$ is diffeomorphic to the boundary $Y_{\Gamma}$ of the four-dimensional plumbing $X_{\Gamma}$ associated to $\Gamma$. Indeed, $X_{\Gamma}$ can be presented as the double branched cover of $D^{4}$ branched along the surface we get by pushing the interior of the surface $F_{\Gamma}$ of the above pluming into $D^{4}$.

If $k$ is a characteristic vector which restricts to a self-conjugate $\operatorname{spin}^{c}$ structure $\mathfrak{s}_{0} \in$ $\operatorname{Spin}^{\mathrm{c}}\left(Y_{\Gamma}\right)$, then the graded root $\left(R_{\Gamma}, w_{k}\right)$ comes with an involution $J: \mathbb{H}^{-}(R, w) \rightarrow$ $\mathbb{H}^{-}(R, w)$, induced by the map

$$
\ell \mapsto-\ell-\frac{1}{2} P D(k)
$$

for $\ell \in H_{2}\left(X_{\Gamma}, \mathbb{Z}\right)$, cf. [4, Sect. 2.3]. We denote its lift to $C(R)$ by $J_{\#}: C\left(R_{\Gamma}\right) \rightarrow$ $C\left(R_{\Gamma}\right)$.

Theorem 5.2 Let $K=K_{\Gamma}$ be an arborescent knot associated to an almost-rational plumbing tree $\Gamma$. Then there exists a chain homotopy equivalence $\left(\mathrm{CF}^{-}\left(\Sigma(K), \mathfrak{s}_{0}\right), \tau_{\#}\right)$ $\simeq\left(C\left(R_{\Gamma}\right), J_{\#}\right)$ of $\iota$-complexes.

Proof Let $k_{0} \in H^{2}\left(X_{\Gamma}, \mathbb{Z}\right)$ be a characteristic vector of the intersection lattice of $X_{\Gamma}$ which restricts to $\mathfrak{s}_{0}$ on the boundary. Theorem 4.3 provides an isomorphism

$$
H_{*}\left(\mathrm{CF}^{-}\left(\Sigma(K), \mathfrak{s}_{0}\right)\right)=\operatorname{HF}^{-}\left(\Sigma(K), \mathfrak{s}_{0}\right) \simeq \mathbb{H}^{-}\left(R_{\Gamma}, w_{k_{0}}\right)=H_{*}\left(C\left(R_{\Gamma}\right)\right) .
$$

As a consequence of Lemma 4.5, the proof that the push-forward of $J: \mathbb{H}^{-}\left(R_{\Gamma}, w_{k_{0}}\right) \rightarrow$ $\mathbb{H}^{-}\left(R_{\Gamma}, w_{k}\right)$ through this isomorphism agrees with $\tau_{*}: \mathrm{HF}^{-}\left(\Sigma(K), \mathfrak{s}_{0}\right) \rightarrow$ $\mathrm{HF}^{-}\left(\Sigma(K), \mathfrak{s}_{0}\right)$ would complete the argument.

Denote by $W_{\Gamma}$ the cobordism $S^{3} \rightarrow Y_{\Gamma}$ obtained from $X_{\Gamma}$ by removing the interior of a small ball $D^{4} \subset X_{\Gamma}$. Proceeding as in the proof of Lemma 4.6, the claim is reduced to the identity

$$
\tau_{\#} \circ F_{W_{\Gamma}, k}^{-}=F_{W_{\Gamma},-k}^{-},
$$

where $k$ is any characteristic vector which restricts to $\mathfrak{s}_{0}$ on the boundary. According to [38] the covering involution $\tau: Y_{\Gamma} \rightarrow Y_{\Gamma}$ extends over $X_{\Gamma}$ as the complex conjugation $T: X_{\Gamma} \rightarrow X_{\Gamma}$. Since $T$ acts on $\operatorname{spin}^{c}$ structures as $\operatorname{spin}^{c}$ conjugation, Lemma 3.6 implies the claimed identity.

Corollary 5.3 Let $K=K_{\Gamma}$ be an arborescent knot associated to an almost-rational plumbing tree $\Gamma$. Then $\bar{\delta}(K)=\delta(K)$ and $\underline{\delta}(K)=-\sigma(K) / 4$, where $\sigma(K)$ denotes the signature of $K$.

Proof As consequence of [4, Theorem 3.1] and Theorem 5.2 above we have an identification $\operatorname{HFB}^{-}(K) \simeq H_{*}\left(\operatorname{Cone}\left(1+J_{\#}\right)\right) \simeq H_{F I}\left(\Sigma(K), \mathfrak{s}_{0}\right)$. Thus $\bar{\delta}(K)=$ 
$\bar{d}\left(\Sigma(K), \mathfrak{s}_{0}\right)$ and $\underline{\delta}(K)=\underline{d}\left(\Sigma(K), \mathfrak{s}_{0}\right)$. Hence [4, Theorem 1.2] implies that the invariant $\bar{\delta}(K)$ agrees with the Ozsváth-Szabó correction term of the double branched cover $d\left(\Sigma(K), \mathfrak{s}_{0}\right)=\delta(K)$. Furthermore, $\underline{\delta}(K)=-2 \cdot \bar{\mu}\left(\Gamma, \mathfrak{s}_{0}\right)$ where $\bar{\mu}\left(\Gamma, \mathfrak{s}_{0}\right)$ denotes the Neumann-Siebenmann $\bar{\mu}$-invariant of the plumbing $\Gamma$ in the spin structure $\mathfrak{s}_{0}$. On the other hand, according to [37, Theorem 5] we have $\sigma(K)=8 \cdot \bar{\mu}\left(\Gamma, \mathfrak{s}_{0}\right)$ thus $\underline{\delta}(K)=-\sigma(K) / 4$.

The connected group associated to the $\iota$-complex $\left(C(R), J_{\#}\right)$ of a graded root $(R, w)$ can be easily computed. Given a vertex $v \in R$ denote by $C(v)$ the set of all leaves of $R$ that are connected to $v$ by an oriented path. We construct a subset $S$ of the leaves of $R$ by the following algorithm. Let $v_{0}$ denote the $J$-invariant vertex $v_{0}$ of $R$ with highest weight. If $C\left(v_{0}\right)$ consists of only one vertex, we add it to $S$; otherwise, we can find a pair $\{v, J v\}$ in $C\left(v_{0}\right)$ and in this case we add both $v$ and $J v$ to $S$. Next consider the path $\gamma$ connecting $v_{0}$ to infinity. Take $v_{1} \in R$ to be the first vertex along $\gamma$ for which $C\left(v_{0}\right) \subsetneq C\left(v_{1}\right)$. If $C\left(v_{1}\right)$ contains a pair of leaves $\{v, J v\}$ with weight larger than the weight of any leaf in $S$ then we choose one such pair with largest possible weight and we add it to $S$. By keep iterating this procedure until $\gamma$ merges with the long stem we end up with a set $S$ of distiguished leaves. We denote by $M$ the smallest graded root $M \subset R$ containing $S$. According to [11, Proposition 7.5] we have that $\operatorname{HFB}_{\text {conn }}^{-}(K)=\mathbb{H}^{-}\left(M,\left.w\right|_{M}\right)$. The resulting $M$ is the monotone subroot of $(R, w)$.

\section{Some independence results}

The following observation is a simple corollary of the concluding statements of Sect. 3.

Corollary 6.1 Suppose that $\mathcal{F}=\left\{K_{i} \mid i \in I\right\}$ is a family of knots with $\operatorname{HFB}_{\text {red-conn }}^{-}(K)=0$ for all $K \in \mathcal{F}$. If $\langle\mathcal{F}\rangle$ denotes the subgroup of $\mathcal{C}$ generated the equivalence classes of $K_{i} \in \mathcal{F}$, then $\operatorname{HFB}_{\text {red-conn }}^{-}(K)=0$ for all $K \in\langle\mathcal{F}\rangle$.

Proof Lemmas 3.8 and 3.9, together with the equivalence provided by Proposition 3.7 imply the result.

Recall the definition of the subgroups $\mathcal{Q} \mathcal{A}$ and $\mathcal{T}$ (generated by quasi-alternating and torus knots, respectively) of the smooth concordance group $\mathcal{C}$ from Sect. 1 .

Proposition 6.2 For $[K] \in \mathcal{Q} \mathcal{A}+\mathcal{T}$ we have that $\operatorname{HFB}_{\text {red-conn }}^{-}(K)=0$.

Proof In Sect. 4 we showed that $\operatorname{HFB}_{\text {red-conn }}^{-}(K)=0$ for $K$ a quasi-alternating knot or a torus knot. Application of Corollary 6.1 concludes the argument.

Based on this proposition, nonvanishing results for $\mathrm{HFB}_{\text {red-conn }}^{-}$then immediately imply nonvanishing in the quotient group $\mathcal{C} /(\mathcal{Q} \mathcal{A}+\mathcal{T})$.

We now prove Theorem 1.6.

Let $C=\left(C_{0}, \partial_{0}, \iota_{0}\right)$ denote the $\iota$-complex where $C_{0}$ (as an $\mathbb{F}[U]$-module) is generated by three generators $a, b, c$, the boundary map $\partial_{0}$ is given by $\partial_{0} a=\partial_{0} b=0$ and $\partial_{0} c=U a+U b$ and $\iota_{0}(a)=b, \iota_{0}(b)=a, \iota_{0}(c)=c$. Define the grading by 
Fig. 3 On the left we give the plumbing graph $\Gamma_{q}$ giving the pretzel knot $P(2,-3,-q)$. On the right the monotone subroot of the associated graded root is shown

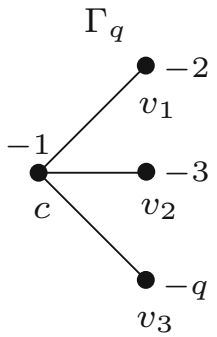

(a)

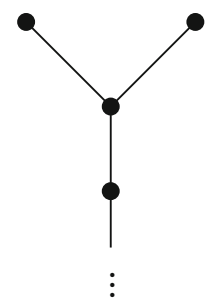

(b)

$\operatorname{gr}(a)=\operatorname{gr}(b)=0$ and $\operatorname{gr}(c)=-1$, and denote by $C[r]$ the same chain complex with grading shifted by $r \in \mathbb{Q}$. (The same chain complex has been denoted by $\mathcal{C}_{1}$ in $[11$, Subsection 7.1].)

Lemma 6.3 Let $q \geq 7$ be any odd integer. Then $\left(\mathrm{CF}^{-}\left(\Sigma(P(2,-3,-q)), \mathfrak{s}_{0}\right), \tau_{\#}\right)$ is locally equivalent to $C[r]$ for some $r$. In particular, $\operatorname{HFB}_{\text {red-conn }}^{-}(P(2,-3,-q))=\mathbb{F}$.

Proof The pretzel knot $-P(-2,3, q)=P(2,-3,-q)$ with $q \geq 7$ is associated to the negative definite star-shaped (hence almost-rational) plumbing tree $\Gamma_{q}$ shown in Figure 3(a). Let us label the vertices of $\Gamma_{q}$ by $c, v_{1}, v_{2}, v_{3}$ as shown by Figure 3(a). (Notice that by successively blowing down $c, v_{1}$ and $v_{2}$ we can see that the threemanifold $Y_{\Gamma_{q}}$ defined by the plumbing is the result of $(-q+6)$-surgery on the righthanded trefoil knot).

In determining the connected Floer homology of these pretzel knots, we will appeal to the computational scheme through lattice homology (as outlined in Sect. 5 for appropriate arborescent knots). Recall that the canonical characteristic vector $K_{q} \in H^{2}\left(X_{\Gamma_{q}} ; \mathbb{Z}\right)$ is the class satisfying $K_{q}(v)=-2-v^{2}$ for each vertex (where a vertex $v$ is viewed as an element of $H_{2}\left(X_{\Gamma_{q}} ; \mathbb{Z}\right)$ and $v^{2}$ denotes the weight on the vertex $v$, which is equal to the self-intersection of the corresponding homology class). In particular, $K_{q}(c)=-1, K_{q}\left(v_{1}\right)=0, K_{q}\left(v_{2}\right)=1$ and $K_{q}\left(v_{3}\right)=q-2$. Every characteristic cohomology element determines a $\operatorname{spin}^{c}$ structure on the boundary three-manifold; by following the blow-down sequence described above with $K_{q}$ we see that it induces the unique spin structure $\mathfrak{s}_{0}$ on the boundary. For an element $x=\alpha c+\beta v_{1}+\gamma v_{2}+\delta v_{3} \in H_{2}\left(X_{\Gamma_{q}} ; \mathbb{Z}\right)$ the quadratic function $\chi_{K_{q}}$ of Equation (7) is given by

$$
2 \chi_{K_{q}}\left(\alpha c+\beta v_{1}+\gamma v_{2}+\delta v_{3}\right)=\alpha^{2}+2 \beta^{2}+3 \gamma^{2}+q \delta^{2}-2 \alpha(\beta+\gamma+\delta)+\alpha-\gamma-(q-2) \delta .
$$

It is not hard to see that $\chi_{K_{q}}(x) \geq 0$ for any $x \in H_{2}\left(X_{\Gamma_{q}} ; \mathbb{Z}\right)$. In order to determine the monotone subroot $M_{q}$ of the graded root $\left(R_{K_{q}}, w_{K_{q}}\right)$ it will be sufficient to understand $S_{0}$ and $S_{1}$ (in the terminology of Subsection 4.4, i.e., $S_{n}=\left\{\chi_{K_{q}} \leq n\right\}$ ). For $q=7$ this calculation has been performed in [29, Subsection 3.2], where it has been shown that $\left\{\chi_{K_{7}} \leq 0\right\}$ has two components, while $\left\{\chi_{K_{7}} \leq 1\right\}$ has a single component. This shows that $M_{7}$ is of the form given by Fig. $3 b$. 
Fig. 4 The plumbing graph $\Delta_{q}$

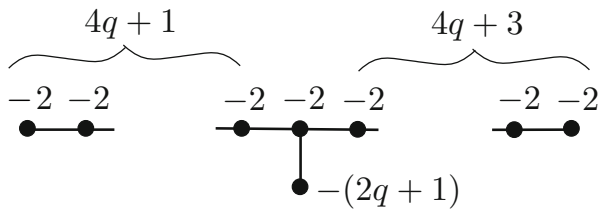

Notice, however, that for $x=\alpha c+\beta v_{1}+\gamma v_{2}+\delta v_{3}$ we have $2 \chi_{K_{q}}(x)=2 \chi_{K_{7}}(x)+$ $(q-7)\left(\delta^{2}-\delta\right)$. Since $\chi_{K_{q}}(x) \geq 0$ for all $x$, we have that $S_{0}$ and $S_{1}$ have the same cardinality for all $q \geq 7$, hence the monotone subroot will be the same for all $q$.

The explicit description of the two vectors representing $S_{0}$ (as given in [29]) and the description of the involution in Eq. (8) now identifies the action of $\tau_{\#}$, verifying the claim about the $\iota$-complex. Then the definition of $\operatorname{HFB}_{\text {conn }}^{-}(P(2,-3,-q))$ shows that in this case it is isomorphic to $\operatorname{HF}^{-}\left(\Sigma(P(2,-3,-q)), \mathfrak{s}_{0}\right)$, and hence that the reduced group $\mathrm{HFB}_{\text {red-conn }}^{-}(P(2,-3,-q))$ has dimension one, completing the proof.

Remark 6.4 Indeed, $\widehat{\mathrm{HF}}(\Sigma(P(2,-3,-q)))$ can be easily computed using the surgery exact triangle (by viewing this three-manifold as surgery along the trefoil knot). This computation shows that $\operatorname{dim} \widehat{\mathrm{HF}}\left(\Sigma(P(2,-3,-q)), \mathfrak{s}_{0}\right)=3$ (and all other $\operatorname{spin}^{c}$ structures have one-dimensional invariant), showing that the local equivalence claimed in Lemma 6.3 is, indeed, a chain homotopy equivalence. Also, the gradings can be easily determined by computing $K_{q}^{2}$. The detailed calculation in the proof of Lemma 6.3 is crucial in the identification of the involution $\tau_{\#}$.

Proof of Theorem 1.6 Suppose that $K$ is the connected sum of pretzel knots $P(2,-3$, $-q$ ) for some $q$ 's (all with $q \geq 7$ ). By [11, Proposition 7.1] the tensor product of these ı-complexes have nonvanishing connected homology, hence the combination of this nonvanishing result with Proposition 6.2 implies that $[K] \in \mathcal{C} /(\mathcal{Q A}+\mathcal{T})$ is nontrivial. In the statement of the theorem we considered mirrors of the knots encountered above; since $\mathrm{HFB}_{\text {red-conn }}^{-}(-K)$ and $\mathrm{HFB}_{\text {red-conn }}^{-}(K)$, as well as $[K]$ and $[-K]$ in $\mathcal{C} /(\mathcal{Q} \mathcal{A}+\mathcal{T})$ are trivial at the same time, the proof is complete.

Next we verify Theorem 1.7 from Sect. 1 . For a knot $K \subset S^{3}$ define $\omega(K)=$ $\min \left\{n \geq 0 \mid U^{n} \cdot \mathrm{HFB}_{\text {red-conn }}^{-}(K)=0\right\}$.

Proof of Theorem 1.7 The double branched cover of the pretzel knot $K_{q}=P(-2 q-$ $1,4 q+1,4 q+3)$ can be expressed as boundary of the negative definite plumbing along the tree $\Delta_{q}$ of Fig. 4 . The associated graded root $R_{q}$ was partially computed in [15]. According to [15] the top part of $R_{q}$, describing the truncated Heegaard Floer group $\mathrm{HF}_{\geq \delta\left(K_{q}\right)-2 q}^{-}\left(\Sigma\left(K_{q}\right)\right)$, looks like the oriented graph of Fig. 5a.

The monotone subroot $M_{q} \subset R_{q}$ of a graded root of this form can be determined as it is explained at the end of Sect. 5, providing the graded roots of Fig. $5 \mathrm{~b}$ (see also [4, Theorem 6.1 and Fig. 4]). This calculation implies

$$
\mathrm{HFB}_{\text {conn }}^{-}\left(K_{q}\right)=\mathbb{F}[U] \oplus \mathbb{F}[U] /\left(U^{q}\right) .
$$


Fig. 5 Schematic picture of the graded root associated to $\Delta_{q}$ in (a) and its monotone subroot in (b). Notice that there is no element of top degree fixed by the reflection

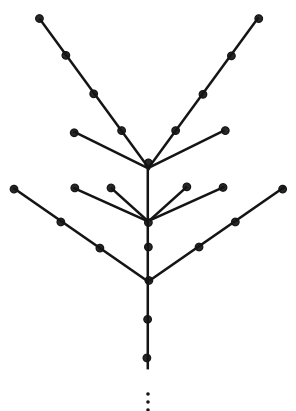

(a)

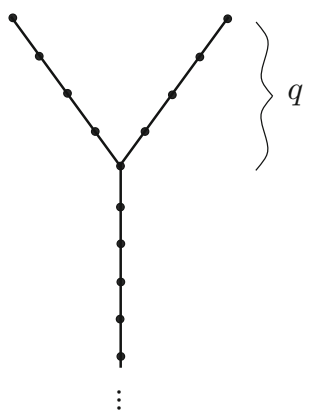

(b)

The application of [11, Proposition 7.1] calculates $\operatorname{HFB}_{\text {conn }}^{-}(K)$ for a knot $K$ that is a positive linear combination of the pretzel knots $K_{q}$ :

$$
\mathrm{HFB}_{\mathrm{conn}}^{-}\left(K_{q_{1}} \# \cdots \# K_{q_{s}}\right)=\mathbb{F}[U] \oplus \bigoplus_{i=1}^{s} \mathbb{F}[U] / U^{q_{i}}
$$

In particular, for a knot of this form we have that

$$
\omega\left(K_{q_{1}} \# \cdots \# K_{q_{s}}\right)=\max _{i} q_{i}
$$

Suppose that there is a non-trivial linear relation among the classes represented by the pretzel knots $K_{q}$ in $\mathcal{C} /(\mathcal{T}+\mathcal{Q} \mathcal{A})$. By grouping the summands of such a linear relation according to their sign we end up with an identity in the knot concordance group $\mathcal{C}$ of the form

$$
K_{a_{1}} \# \ldots \# K_{a_{s}}=K_{b_{1}} \# \ldots \# K_{b_{l}} \# P
$$

for some $P \in \mathcal{T}+\mathcal{Q} \mathcal{A}$. Without loss of generality we can assume that the relation is reduced, i.e. that $a_{i} \neq b_{j}$ for each $i$ and $j$. On the other hand, as a consequence of Equation (9) we have that

$$
\max _{i} a_{i}=\omega\left(K_{a_{1}} \# \cdots \# K_{a_{s}}\right)=\omega\left(K_{b_{1}} \# \ldots \# K_{b_{l}} \# P\right)=\omega\left(K_{b_{1}} \# \cdots \# K_{b_{l}}\right)=\max _{j} b_{j}
$$

a contradiction. This shows that the subgroup $\left\langle\left[K_{q}\right]\right| q$ odd and $\left.q \geq 7\right\rangle \subset \mathcal{C} /(\mathcal{T}+$ $\mathcal{Q} \mathcal{A})$ generated by the above pretzel knots is isomorphic to $\mathbb{Z}^{\infty} \subset \mathcal{C} /(\mathcal{T}+\mathcal{Q} \mathcal{A})$.

In [3] Dai, Hom, Stoffregen, and Truong showed that the Brieskorn spheres $\Sigma(2 q+$ $1,4 q+1,4 q+3)(q \geq 1)$ generate a $\mathbb{Z}^{\infty}$ summand of the homology cobordism group $\Theta_{\mathbb{Z}}^{3}$. This was done by constructing a family $\left\{\phi_{n}\right\}$ of homomorphisms from the group of almost $\iota$-complexes to $\mathbb{Z}$, and then proving that the given Brieskorn spheres are mapped to 1 for exactly one of the homomorphisms and mapped to 0 for all the others. The same argument applies in our case, and thus the $\mathbb{Z}^{\infty}$ subgroup of $\mathcal{C} /(\mathcal{T}+\mathcal{Q} \mathcal{A})$, 
generated by the pretzel knots $K_{q}=P(-2 q-1,4 q+1,4 q+3)(q \geq 1)$, is indeed a direct summand, concluding the proof.

Acknowledgements Open access funding provided by Alfréd Rényi Institute of Mathematics.

Open Access This article is licensed under a Creative Commons Attribution 4.0 International License, which permits use, sharing, adaptation, distribution and reproduction in any medium or format, as long as you give appropriate credit to the original author(s) and the source, provide a link to the Creative Commons licence, and indicate if changes were made. The images or other third party material in this article are included in the article's Creative Commons licence, unless indicated otherwise in a credit line to the material. If material is not included in the article's Creative Commons licence and your intended use is not permitted by statutory regulation or exceeds the permitted use, you will need to obtain permission directly from the copyright holder. To view a copy of this licence, visit http://creativecommons.org/licenses/by/4.0/.

\section{References}

1. Alfieri, A.: Upsilon-type concordance invariants. Algebr. Geom. Topol. 19(7), 3315-3334 (2019)

2. Dai, I., Hedden, M., Mallick, A.: Personal communication. (2019)

3. Dai, I., Hom, J., Stoffregen, M., Truong, L.: An infinite-rank summand of the homology cobordism group. arXiv: 1810.06145, (2018)

4. Dai, I., Manolescu, C.: Involutive Heegaard Floer homology and plumbed three-manifolds. J. Inst. Math. Jussieu 18(6), 1115-1155 (2019)

5. Eisenbud, D., Neumann, W.: Three-dimensional Link Theory and Invariants of Plane Curve Singularities vol. 110 of Annals of Mathematics Studies. Princeton University Press, Princeton (1985)

6. Gabai, D.: Genera of the arborescent links. Mem. Am. Math. Soc. 59(339), 1-98 (1986). (i-vii)

7. Gompf, R., Stipsicz, A.: 4-Manifolds and Kirby Calculus. Graduate Studies in Mathematics, vol. 20. American Mathematical Society, Providence (1999)

8. Greene, J., Jabuka, S.: The slice-ribbon conjecture for 3-stranded pretzel knots. Am. J. Math. 133, 555-580 (2011)

9. Grigsby, E.: Knot Floer homology in cyclic branched covers. Algebr. Geom. Topol. 6(3), 1355-1398 (2006)

10. Grigsby, E., Ruberman, D., Strle, S.: Knot concordance and Heegaard Floer homology invariants in branched covers. Geom. Topol. 12(4), 2249-2275 (2008)

11. Hendricks, K., Hom, J., Lidman, T.: Applications of involutive Heegaard Floer homology. arXiv: $1802.02008,(2018)$

12. Hendricks, K., Lipshitz, R., Sarkar, S.: A flexible construction of equivariant Floer homology and applications. J. Topol. 9(4), 1153-1236 (2016)

13. Hendricks, K., Manolescu, C.: Involutive Heegaard Floer homology. Duke Math. J. 166(7), 1211-1299 (2017)

14. Hendricks, K., Manolescu, C., Zemke, I.: A connected sum formula for involutive Heegaard Floer homology. Selecta Math. (N.S.) 24(2), 1183-1245 (2018)

15. Hom, J., Karakurt, C., Lidman, T.: Surgery obstructions and Heegaard Floer homology. Geom. Topol. 20(4), 2219-2251 (2016)

16. Juhász, A., Thurston, D., Zemke, I.: Naturality and mapping class groups in Heegaard Floer homology. arXiv:1210.4996, (2018)

17. Lecuona, A.: On the slice-ribbon conjecture for pretzel knots. Algebr. Geom. Topol. 15(4), 2133-2173 (2015)

18. Levine, A.: Computing knot Floer homology in cyclic branched covers. Algebr. Geom. Topol. 8(2), $1163-1190$ (2008)

19. Lewark, L., Lobb, A.: Upsilon-like concordance invariants from $\mathfrak{s l}_{n}$ knot cohomology. Geom. Topol. 23(2), 745-780 (2019)

20. Lidman, T., Moore, A.: Pretzel knots with $L$-space surgeries. Mich. Math. J. 65(1), 105-130 (2016)

21. Lisca, P.: Lens spaces, rational balls and the ribbon conjecture. Geom. Topol. 11, 429-472 (2007)

22. Manolescu, C., Owens, R.: A concordance invariant from the Floer homology of double branched covers. Int. Math. Res. Not. IMRN 20, 21 (2007). (Art. ID rnm077) 
23. Némethi, A.: Lattice cohomology of normal surface singularities. Publ. RIMS. Kyoto Univ. 44, 507$543(2008)$

24. Némethi, A.: Links of rational singularities, $L$-spaces and LO fundamental groups. Invent. Math. 210(1), 69-83 (2017)

25. Ozsváth, P., Stipsicz, A., Szabó, Z.: Knots in lattice homology. Comment. Math. Helv. 89(4), 783-818 (2014)

26. Ozsváth, P., Stipsicz, A., Szabó, Z.: A spectral sequence on lattice homology. Quantum Topol. 5(4), 487-521 (2014)

27. Ozsváth, P., Stipsicz, A., Szabó, Z.: Concordance homomorphisms from knot Floer homology. Adv. Math. 315, 366-426 (2017)

28. Ozsváth, P., Szabó, Z.: Absolutely graded Floer homologies and intersection forms for four-manifolds with boundary. Adv. Math. 173(2), 179-261 (2003)

29. Ozsváth, P., Szabó, Z.: On the Floer homology of plumbed three-manifolds. Geom. Topol. 7, 185-224 (2003)

30. Ozsváth, P., Szabó, Z.: Holomorphic disks and three manifold invariants: properties and applications. Ann. Math. 159, 1159-1245 (2004)

31. Ozsváth, P., Szabó, Z.: Holomorphic disks and topological invariants for closed three manifolds. Ann. Math. 159, 1027-1158 (2004)

32. Ozsváth, P., Szabó, Z.: An introduction to Heegaard Floer homology. In: Floer Homology, Gauge Theory, and Low Dimensional Topology, Proceedings of the Clay Mathematics Institute, (2004)

33. Ozsváth, P., Szabó, Z.: Lectures on Heegaard Floer homology. In: Floer Homology, Gauge Theory, and Low Dimensional Topology, Proceedings of the Clay Mathematics Institute, (2004)

34. Ozsváth, P., Szabó, Z.: Holomorphic triangles and invariants for smooth four-manifolds. Adv. Math. 202, 326-400 (2006)

35. Perutz, T.: Hamiltonian handleslides for Heegaard Floer homology. In: Proceedings of Gökova Geometry-Topology Conference 2007, pages 15-35. Gökova Geometry/Topology Conference (GGT), Gökova, (2008)

36. Piccirillo, L.: The Conway knot is not slice. arXiv:1808.02923 (2018)

37. Saveliev, N.: A surgery formula for the $\bar{\mu}$-invariant. Topol. Appl. 106(1), 91-102 (2000)

38. Saveliev, N.: Invariants for homology 3-spheres, volume 140 of Encyclopaedia of Mathematical Sciences. In: Low-Dimensional Topology, I. Springer-Verlag, Berlin (2002)

39. Wang, S.: Semigroups of $L$-space knots and nonalgebraic iterated torus knots. Math. Res. Lett. 25(1), 335-346 (2018)

40. Yozgyur, R.: Knots not concordant to $l$-space knots. Periodica Math. Hungarica, to appear, arXiv:1905.02461 (2019)

41. Zemke, I.: Graph cobordisms and Heegaard Floer homology. arXiv:1512.01184, (2015)

Publisher's Note Springer Nature remains neutral with regard to jurisdictional claims in published maps and institutional affiliations. 